\title{
HOMOTOPY THEORY OF MODULES AND GORENSTEIN RINGS
}

\author{
APOSTOLOS BELIGIANNIS
}

\section{Introduction}

It is an old observation of Eckmann-Hilton [21], that the homotopy theory of topological spaces has an algebraic analogue in the module category of a ring. Inspired by the work of Eckmann-Hilton, various authors studied the problem of constructing a homotopy theory in more general algebraic categories. We refer to the works of Heller [18], [20], [19], Huber [23], Kleisli [29], Brown [12], Auslander-Bridger [1] and Quillen [35]. Restricting to the case of a module category, there are two different in general, homotopy theories defined. The injective homotopy which is defined by killing the injective modules and the projective homotopy which is defined by killing the projective modules. Let $\Lambda$ be an associative ring, and let $\operatorname{Mod}(\Lambda)$ be the category of right $\Lambda$-modules. Using injective homotopy we obtain the stable category $\overline{\operatorname{Mod}}(\Lambda)$ which is always right triangulated, and using projective homotopy we obtain the stable category $\underline{\operatorname{Mod}}(\Lambda)$ which is always left triangulated. The projective and the injective homotopy coincide iff the ring is Quasi-Frobenius (QF-ring for short) and in this case the stable category $\underline{\operatorname{Mod}}(\Lambda)=\overline{\operatorname{Mod}}(\Lambda)$ is a compactly generated triangulated category. The stable module category of a modular group algebra (which is a QF-ring), has been studied by many authors mainly from the representation theoretic point of view. There is recently a big progress in this study, which is developed using machinery from the theory of triangulated categories, in particular Bousfield's localization techniques, see for example [36], [11]. Our main purpose in this paper is to study the stable module categories $\underline{\operatorname{Mod}}(\Lambda)$ and $\overline{\operatorname{Mod}}(\Lambda)$ of a ring $\Lambda$ from the point of view of modern algebraic homotopy theory. This is possible if the stable module categories are compactly generated, so a theory of Brown Representability can be developed.

The paper is organized as follows.

In Section 2, we study general stable categories with products or coproducts which are induced by homologically finite subcategories in the sense of Auslander-Smalø [2]. In Section 3 we study Brown Representability and its

Received October 6, 1998. 
consequences, in a given additive category with coproducts and weak cokernels. Here the notion of a Whitehead subcategory borrowed from topology is of central importance. Brown's Theorem in this setting has interesting applications to (right) triangulated categories, to $t$-structures in the sense of [4] and to locally finitely presented additive categories in the sense of [13]. In particular we show that Bousfield's localization holds in a compactly generated right triangulated category. In Section 4 we prove that a functorially finite subcategory $\mathscr{X}$ of an additive category $\mathscr{C}$, induces a closed model structure on $\mathscr{C}$ in the sense of Quillen [35], with all objects fibrant and cofibrant. Conversely any such closed model structure arises from a functorially finite subcategory. The associated homotopy category is the stable category $\mathscr{C} / \mathscr{X}$ which in many cases has the structure of a pre-triangulated category, i.e. it is left and right triangulated in a compatible way.

Section 5 is devoted to the characterization of rings, such that the stable categories modulo projectives or injectives are "suitable" for doing homotopy theory. For the injective homotopy this happens iff the ring $\Lambda$ is right Morita. For the projective homotopy this happens iff the ring $\Lambda$ is left coherent and right perfect. In both cases the stable categories are compactly generated Abstract Homotopy Categories [12], and Brown Representability Theorem holds in this setting. Note that our results on injective homotopy generalize recent results of Jørgensen [25].

In Section 6, inspired from the construction of the stable homotopy category of spectra [32], we study the existence of a stable homotopy category associated to the projective or injective homotopy of a ring $\Lambda$. Since the stable module categories are not in general triangulated, it is useful in many cases to replace them by their stabilizations [8], [19], which are triangulated categories, and this can be done in a universal way. We say that a ring $\Lambda$ has a projective, resp. injective, stable homotopy category if the stabilization of $\underline{\operatorname{Mod}}(\Lambda)$, resp. of $\overline{\operatorname{Mod}}(\Lambda)$, is compactly generated. We prove that in case $\Lambda$ is right Gorenstein in the sense of [8], and the ring $\Lambda$ is left coherent and right perfect or right Morita, then such a stable homotopy category exists and can be described as the triangulated stable category of Cohen-Macaulay modules. We close the paper studying when the stable homotopy category is a phantomless or Brown category in the sense of [6], [7].

Throughout this paper we compose morphisms in the diagrammatic order, i.e. the composition of $f: A \rightarrow B, g: B \rightarrow C$ is denoted by $f \circ g$. 


\section{Stable Categories with Coproducts and Compact Objects}

We fix in this section an additive category $\mathscr{C}$ with split idempotents and a full additive subcategory $\mathscr{X} \subseteq \mathscr{C}$ of $\mathscr{C}$ closed under isomorphisms and direct summands. A morphism $f: A \rightarrow B$ in $\mathscr{C}$ is called an $\mathscr{X}$-epic if the morphism $\mathscr{C}(\mathscr{X}, f): \mathscr{C}(\mathscr{X}, A) \rightarrow \mathscr{C}(\mathscr{X}, B)$ is surjective. We recall from [2] that the subcategory $\mathscr{X}$ is called contravariantly finite in $\mathscr{C}$ if for any object $A \in \mathscr{C}$, there exists an $\mathscr{X}$-epic $\chi_{A}: X_{A} \rightarrow A$ with $X_{A} \in \mathscr{X}$. The morphism $\chi_{A}$ is called a right $\mathscr{X}$-approximation of $A$. The notions of an $\mathscr{X}$-monic morphism and of a covariantly finite subcategory are defined dually. A subcategory is called functorially finite if it is both covariantly and contravariantly finite.

We denote the stable category of $\mathscr{C}$ modulo $\mathscr{X}$ by $\mathscr{C} / \mathscr{X}$. We recall that the objects of $\mathscr{C} \mid \mathscr{X}$ are the objects of $\mathscr{C}$. If $A, B$ are objects of $\mathscr{C} \mid \mathscr{X}$, then $\mathscr{C} / \mathscr{X}(A, B)=\mathscr{C}(A, B) / \mathscr{I}_{\mathscr{X}}(A, B)$, where $\mathscr{I}_{\mathscr{X}}(A, B)$ is the subgroup of $\mathscr{C}(A, B)$ consisting of all morphisms factorizing through an object of $\mathscr{X}$. We denote by $\underline{A}$ the object $A$ considered as an object of $\mathscr{C} / \mathscr{X}$ and by $f$ the class of the morphism $f: A \rightarrow B$ in $\mathscr{C} \mid \mathscr{X}(A, B)$. Then $\mathscr{C} \mid \mathscr{X}$ is an additive category and setting $\pi(A)=\underline{A}$ and $\pi(f)=\underline{f}$, we obtain the projection functor $\pi: \mathscr{C} \rightarrow \mathscr{C} / \mathscr{X}$.

Proposition 2.1. Suppose that $\mathscr{C}$ has coproducts (products).

(1) The functor $\pi: \mathscr{C} \rightarrow \mathscr{C} / \mathscr{X}$ preserves coproducts (products) $\Leftrightarrow \mathscr{X}$ is closed under coproducts (products). In this case $\mathscr{C} / \mathscr{X}$ has coproducts (products).

(2) If $\mathscr{X}$ is contravariantly finite (covariantly finite), then $\mathscr{X}$ is closed under coproducts (products).

(3) If $\mathscr{X}$ is closed under coproducts (products), then idempotents split in $\mathscr{C} \mid \mathscr{X}$.

Proof. (1) Assume first that $\mathscr{X}$ is closed under coproducts. Let $\left\{\underline{A}_{i} ; i \in I\right\}$ be a set of objects in $\mathscr{C} / \mathscr{X}$ and let $\mu_{i}: A_{i} \rightarrow \oplus A_{i}$ be the injections into the coproduct in $\mathscr{C}$. Then we have morphisms $\underline{\mu}_{i}: \underline{A}_{i} \rightarrow \oplus A_{i}$ in $\mathscr{C} / \mathscr{X}$. Let $\underline{f}_{i}: \underline{A}_{i} \rightarrow \underline{B}$ be morphisms in $\mathscr{C} / \mathscr{X}$. Choose morphisms $f_{i}^{\prime}: A_{i} \rightarrow$ $B$ in $\mathscr{C}$, such that $\underline{f}_{i}^{\prime}=\underline{f}_{i}, \forall i \in I$. Then there exists a unique morphism $f^{\prime}: \oplus A_{i} \rightarrow B$ such that $\mu_{i} \circ f^{\prime}=f_{i}^{\prime}, \forall i \in I$. Then in $\mathscr{C} / \mathscr{X}$ we have $\underline{\mu}_{i} \circ \underline{f}^{\prime}=\underline{f}_{i}, \forall i \in I$. Let $\underline{h}: \oplus A_{i} \rightarrow \underline{B}$ be another morphism in $\mathscr{C} / \mathscr{X}$ such that $\underline{\mu}_{i} \circ \underline{h}=\underline{f}_{i}, \forall i \in I$. Then there are morphisms $\kappa_{i}: A_{i} \rightarrow X_{i}$ and $\chi_{i}: X_{i} \rightarrow B$, where $X_{i} \in \mathscr{X}$, such that $\mu_{i} \circ f^{\prime}-\mu_{i} \circ h^{\prime}=\kappa_{i} \circ \chi_{i}$, where $h^{\prime}: \oplus A_{i} \rightarrow B$ is a morphism in $\mathscr{C}$ with $\underline{h}^{\prime}=\underline{h}$. Let $\oplus \kappa_{i}: \oplus A_{i} \rightarrow \oplus X_{i}$ be the unique morphism with $\mu_{i} \circ \oplus \kappa_{i}=\kappa_{i} \circ v_{i}$ and let $\chi: \oplus X_{i} \rightarrow B$ be the unique morphism with $v_{i} \circ \chi=\chi_{i}$, where $v_{i}: X_{i} \rightarrow \oplus X_{i}$ are the canonical injections. Then $\mu_{i} \circ f^{\prime}-\mu_{i} \circ h^{\prime}=\kappa_{i} \circ \chi_{i}=\kappa_{i} \circ v_{i} \circ \chi=\mu_{i} \circ \oplus \kappa_{i} \circ \chi$. This 
implies that $f^{\prime}-h^{\prime}=\oplus \kappa_{i} \circ \chi$. By hypothesis, $\oplus X_{i}$ is in $\mathscr{X}$ and this implies that $f=f^{\prime}=\underline{h}^{\prime}=\underline{h}$ in $\mathscr{C} / \mathscr{X}$. This shows that $\mathscr{C} / \mathscr{X}$ has and $\pi$ preserves coproducts. Conversely if $\pi$ preserves coproducts, let $\left\{X_{i}, i \in I\right\}$ be a set of objects of $\mathscr{X}$. Then $\pi\left(\oplus X_{i}\right)=\oplus \pi\left(X_{i}\right)=0$ and this implies that $\oplus X_{i}$ is in $\mathscr{X}$. Hence $\mathscr{X}$ is closed under coproducts. The parenthetical case is similar.

(2) Let $X_{i}, i \in I$ be a set of objects in $\mathscr{X}$ and let $\chi: X \rightarrow \oplus X_{i}$ be a right $\mathscr{X}$-approximation of $\oplus X_{i}$. If $\mu_{i}: X_{i} \rightarrow \oplus X_{i}$ are the injections, there are morphisms $\alpha_{i}: X_{i} \rightarrow X$ such that $\alpha_{i} \circ \chi=\mu_{i}$. Then there exists a unique morphism $\psi: \oplus X_{i} \rightarrow X$ such that $\mu_{i} \circ \psi=\alpha_{i}$, hence $\mu_{i} \circ \psi \circ \chi=\mu_{i}$. Then $\psi \circ \chi=1_{\oplus X_{i}}$. Since $\mathscr{X}$ is closed under direct summands, we have $\oplus X_{i} \in \mathscr{X}$. Hence $\mathscr{X}$ is closed under coproducts. Part (3) follows from [16].

If $\mathscr{Y}$ is a full subcategory of $\mathscr{C}$, then add $(\mathscr{Y})$ denotes the full subcategory of $\mathscr{C}$ consisting of all direct summands of finite coproducts of objects of $\mathscr{Y}$. If $\mathscr{C}$ has coproducts, resp. products, then $\operatorname{Add}(\mathscr{Y})$, resp. $\operatorname{Prod}(\mathscr{Y})$, denotes the full subcategory of $\mathscr{C}$ consisting of all direct summands of arbitrary coproducts, resp. products, of objects of $\mathscr{Y}$. Trivially a morphism $f: A \rightarrow B$ is $\mathscr{Y}$-epic (resp. $\mathscr{Y}$-monic) $\Leftrightarrow f$ is $\operatorname{Add}(\mathscr{Y})$-epic, (resp. $\operatorname{Prod}(\mathscr{Y})$-monic).

LEMMA 2.2. If $\mathscr{C}$ has coproducts, resp. products, and $\mathscr{X}$ is skeletally small, then $\operatorname{Add}(\mathscr{X})$, resp. $\operatorname{Prod}(\mathscr{X})$, is contravariantly finite, resp. covariantly $f$ nite, in $\mathscr{C}$.

Proof. For any $C \in \mathscr{C}$ let $I_{C}:=\{X \rightarrow C: X \in \operatorname{Iso}(\mathscr{X})\}$, where $\operatorname{Iso}(\mathscr{X})$ is the set of isoclasses of objects of $\mathscr{X}$ and set $X_{C}:=\bigoplus_{i \in I_{C}} X_{i}$. The set of morphisms $I_{C}$ induces a canonical morphism $\chi_{C}: X_{C} \rightarrow C$, which obviously is a right $\operatorname{Add}(\mathscr{X})$-approximation of $C$. The parenthetical case is dual.

Definition 2.3. ([33]) An object $A$ in an additive category $\mathscr{C}$ is called (countably) compact if the functor $\mathscr{C}(A,-): \mathscr{C} \rightarrow \mathscr{A} b$ preserves (countable) coproducts. A full subcategory $\mathscr{X}$ of $\mathscr{C}$ is called (countably) compact if $\mathscr{X}$ consists of (countably) compact objects.

The full subcategory of $\mathscr{C}$ consisting of all compact objects is denoted by $\mathscr{C}^{b}$. If $\mathscr{C}$ has coproducts, then it is well known that $A \in \mathscr{C}$ is compact iff any morphism $f: A \rightarrow \bigoplus_{i \in I} C_{i}$ factors through a finite subcoproduct $\bigoplus_{j \in J \subseteq I} C_{j},|J|<\infty$. Obviously if $\mathscr{C}$ has coproducts and $\mathscr{X} \subseteq \mathscr{C}^{b}$, then $(\mathscr{C} / \mathscr{X})^{b}=\mathscr{C}^{b} / \mathscr{X} \approx(\mathscr{C} / \operatorname{Add}(\mathscr{X}))^{b}$.

Definition 2.4. ([13]) An object $A$ in an additive category $\mathscr{C}$ is called finitely presented if the functor $\mathscr{C}(A,-): \mathscr{C} \rightarrow \mathscr{A} b$ preserves (filtered) direct limits. We denote by f.p. $(\mathscr{C})$ the full subcategory of $\mathscr{C}$ consisting of all finitely presented objects. An additive category $\mathscr{C}$ is called locally finitely presented 
if $\mathscr{C}$ has (filtered) direct limits, any object of $\mathscr{C}$ is a direct limit of finitely presented objects and finally if f.p. $(\mathscr{C})$ is skeletally small.

Let $\mathscr{X}$ be a skeletally small additive category. We denote by $\operatorname{Mod}(\mathscr{X})$, the Grothendieck category of contravariant additive functors from $\mathscr{X}$ to the category $\mathscr{A} b$ of abelian groups. The full subcategory of projective, resp. flat, functors is denoted by $\operatorname{Proj}(\operatorname{Mod}(\mathscr{X}))$, resp. $\operatorname{Flat}(\operatorname{Mod}(\mathscr{X}))$. By [13], Flat $(\operatorname{Mod}(\mathscr{X}))$ is locally finitely presented and any locally finitely presented category is of this form. For the concept of pure semisimplicity in these categories we refer to [13]; in particular $\mathscr{C}$ is pure semisimple iff $\mathscr{C}=\operatorname{Add}($ f.p. $(\mathscr{C})$ ). For latter use we prove the following.

Proposition 2.5. Let $\mathscr{C}$ be an additive category with products and coproducts and let $\mathscr{X}$ be a skeletally small full subcategory of $\mathscr{C}$ with $\mathscr{X} \subseteq \mathscr{C}^{b}$. Then the following are equivalent.

(i) $\operatorname{Add}(\mathscr{X})$ is covariantly finite in $\mathscr{C}$.

(ii) $\operatorname{Add}(\mathscr{X})$ is a locally finitely presented pure semisimple category with products.

Proof. (i) $\Rightarrow$ (ii) Consider the restriction functor $\mathrm{S}: \mathscr{C} \rightarrow \operatorname{Mod}(\mathscr{X})$ defined by $\mathrm{S}(A)=\left.\mathscr{C}(-, A)\right|_{\mathscr{X}}$. It is easy to see that $\mathrm{S}$ preserves products and coproducts and induces an equivalence between $\operatorname{Add}(\mathscr{X})$ and $\operatorname{Proj}(\operatorname{Mod}(\mathscr{X}))$. If $\operatorname{Add}(\mathscr{X})$ is covariantly finite, then obviously $\operatorname{Add}(\mathscr{X})$ is closed under products in $\mathscr{C}$. Hence $\operatorname{Proj}(\operatorname{Mod}(\mathscr{X}))$ is closed under products in $\operatorname{Mod}(\mathscr{X})$. By Chase's Theorem (see [5]), we have that $\operatorname{Mod}(\mathscr{X})$ is perfect. In particular $\operatorname{Proj}(\operatorname{Mod}(\mathscr{X}))$, hence $\operatorname{Add}(\mathscr{X})$, is equivalent to Flat $(\operatorname{Mod}(\mathscr{X}))$, which by construction is pure semisimple.

(ii) $\Rightarrow$ (i) By hypothesis for any set $\left\{X_{i} ; i \in I\right\}$ of objects of $\mathscr{X}$, the product $\prod X_{i}$ is in $\operatorname{Add}(\mathscr{X})$ and the pure mono $\mu: \oplus X_{i} \rightarrow \prod X_{i}$ splits. If $A \in \mathscr{C}$, then since $\operatorname{Prod}(\mathscr{X})$ is covariantly finite, there exists a left $\operatorname{Prod}(\mathscr{X})$-approximation $f: A \rightarrow \prod_{A \rightarrow X} X$ of $A$ as in Lemma 2.2. If $g: A \rightarrow M$ is a morphism with $M$ is in $\operatorname{Add}(\mathscr{X})$, then there exists a family of $\left\{X_{i} ; i \in I\right\}$ of objects of $\mathscr{X}$ such that $M \oplus N=\oplus X_{i}$. Let $i_{M}: M \rightarrow \oplus X_{i}$ and $p_{M}: \oplus X_{i} \rightarrow M$ be the canonical injection and projection. Also let $v: \prod X_{i} \rightarrow \oplus X_{i}$ be a splitting of the canonical morphism $\mu: \oplus X_{i} \rightarrow \prod X_{i}$. Then by the covariant finiteness of $\operatorname{Prod}(\mathscr{X})$, there exists a morphism $\alpha: \prod_{A \rightarrow X} X \rightarrow \prod X_{i}$ such that $f \circ \alpha=g \circ i_{M} \circ \mu$. Then $f \circ \alpha \circ v=g \circ i_{M} \circ \mu \circ v=g \circ i_{M}$. Hence $f \circ \alpha \circ v \circ p_{M}=g$. This shows that $f$ is a left $\operatorname{Add}(\mathscr{X})$-approximation of $A$. Hence $\operatorname{Add}(\mathscr{X})$ is covariantly finite in $\mathscr{C}$.

From (the proof of) Proposition 2.5 we deduce directly the following.

COROLlary 2.6. Let $\mathscr{C}$ be a locally finitely presented category with products. Then $\operatorname{Add}(\mathrm{f} . \mathrm{p} .(\mathscr{C}))$ is covariantly finite in $\mathscr{C}$ iff $\mathscr{C}$ is pure semisimple. 


\section{Weak Colimits and Brown Representability}

\subsection{Weak Limits and Weak Colimits}

Let $\mathscr{C}$ be an additive category and let $\mathscr{I}: I \rightarrow \mathscr{C}$ be a functor from a small category $I$. We use the notations: $A_{i}=\mathscr{I}(i)$ for $i \in \mathscr{I}$ and $\alpha_{i j}=\mathscr{I}(i \rightarrow j)$ : $A_{i} \rightarrow A_{j}$ for an arrow $i \rightarrow j$ in $I$. We recall that a weak colimit $\mathrm{w} . \lim _{\rightarrow} A_{i}$ of the functor $\mathscr{I}$ is defined as the colimit except the uniqueness property. A weak colimit of a functor $\mathscr{I}: I \rightarrow \mathscr{C}$ is called a weak direct limit, if the category $I$ is filtered. An example of a (finite) weak colimit is a weak cokernel of a morphism $f: A \rightarrow B$ in $\mathscr{C}$, which is defined as a morphism $g: B \rightarrow C$ with $f \circ g=0$ and such that any other morphism $h: B \rightarrow D$ with $f \circ h=0$ factors through $g$ (in a not necessarily unique way). A weak colimit is not uniquely determined and in case $\mathscr{C}$ has coproducts and weak cokernels, then a weak colimit of the functor $\mathscr{I}$ can be obtained as a weak cokernel $\theta_{\mathscr{I}}: \bigoplus_{i \in I} A_{i} \rightarrow$ w. $\lim _{\rightarrow} A_{i}$ of the canonical morphism $\zeta_{\mathscr{I}}: \bigoplus_{i \rightarrow j \in I^{2}} A_{i} \rightarrow \bigoplus_{k \in I} A_{k}$, where $I^{2} \overrightarrow{\text { is }}$ the category of morphisms of $I$. Hence if $\mathscr{C}$ has coproducts and weak cokernels, then $\mathscr{C}$ has weak colimits. We leave to the reader to formulate the dual notions of weak limit and weak kernel. We note that if $\mathscr{C}$ has products and weak kernels, then $\mathscr{C}$ has weak limits.

Proposition 3.1. (1) If $\mathscr{C}$ has weak kernels (weak cokernels) and if $\mathscr{X}$ is a full additive contravariantly finite (covariantly finite) subcategory of $\mathscr{C}$, then $\mathscr{X}, \mathscr{C} / \mathscr{X}$ have weak kernels (weak cokernels). Moreover the canonical functor $\pi: \mathscr{C} \rightarrow \mathscr{C} / \mathscr{X}$ preserves weak kernels (weak cokernels) of $\mathscr{X}$-epics $(\mathscr{X}$-monics).

(2) If $\mathscr{C}$ has products and weak kernels and if $\mathscr{X}$ is contravariantly finite and closed under products in $\mathscr{C}$, then $\mathscr{X}, \mathscr{C} / \mathscr{X}$ have weak limits.

(3) If $\mathscr{C}$ has coproducts and weak cokernels and if $\mathscr{X}$ is covariantly finite and closed under coproducts in $\mathscr{C}$, then $\mathscr{X}, \mathscr{C} \mid \mathscr{X}$ have weak colimits.

Proof. (1) Suppose that $\mathscr{X}$ is contravariantly finite in $\mathscr{C}$ and $\mathscr{C}$ has weak kernels. Let $f: Y \rightarrow Z$ be a morphism in $\mathscr{X}$ with weak kernel $g: A \rightarrow Y$ in $\mathscr{C}$. If $\chi_{A}: X_{A} \rightarrow A$ is a right $\mathscr{X}$-approximation of $A$, then trivially the morphism $\chi_{A} \circ g: X_{A} \rightarrow Y$ is a weak kernel of $f$ in $\mathscr{X}$. Now let $f: \underline{A} \rightarrow \underline{B}$ be a morphism in $\mathscr{C} / \mathscr{X}$ and consider the morphism ${ }^{t}\left(f, \chi_{B}\right): A \oplus \bar{X}_{B} \rightarrow B$ in $\mathscr{C}$. Let $(g, k): C \rightarrow A \oplus X_{B}$ be a weak kernel of ${ }^{t}\left(f, \chi_{B}\right)$ in $\mathscr{C}$ and consider the morphism $g: \underline{C} \rightarrow \underline{A}$ in $\mathscr{C} / \mathscr{X}$. Obviously $g \circ f=0$. If $\underline{h}: \underline{D} \rightarrow \underline{A}$ is a morphism with $\underline{h} \circ \underline{f}=0$, then we have a factorization $h \circ f=m \circ \chi_{B}$, where $m: D \rightarrow X_{B}$. Then $(h,-m) \circ{ }^{t}\left(f, \chi_{B}\right)=0$, hence there exists a morphism $d: D \rightarrow C$ with $d \circ(h,-m)=(g, k)$. Then $d \circ h=g$ and $\underline{d} \circ \underline{h}=\underline{g}$. This means that $\underline{g}$ is a weak kernel of $\underline{f}$ in $\mathscr{C} / \mathscr{X}$. Finally let $f: B \rightarrow \bar{C}$ be an 
$\mathscr{X}$-epic and let $g: A \rightarrow B$ be a weak kernel of $f$. Then in $\mathscr{C} / \mathscr{X}$ we have $g \circ f=0$. Let $\underline{h}: \underline{D} \rightarrow \underline{B}$ be a morphism with $\underline{h} \circ f=0$. Then there exists a morphism $t: D \rightarrow X_{C}$ with $h \circ f=t \circ \chi_{C}$, where $\chi_{C}: X_{C} \rightarrow C$ is a right $\mathscr{X}$-approximation of $C$. Since $f$ is $\mathscr{X}$-epic, there exists a morphism $r: X_{C} \rightarrow B$ with $\chi_{C} \circ r=f$. Then $h \circ f=t \circ r \circ f$, hence $h-t \circ r=k \circ g$, for a morphism $k: D \rightarrow A$. But then $\underline{h}=\underline{k} \circ g$ and this means that $g$ is a weak kernel of $f$ in $\mathscr{C} / \mathscr{X}$. The parenthetical case is dual. Parts (2), ( $\overline{3})$ are consequences of (1) and Proposition 2.1.

Definition 3.2. Let $\mathscr{P}$ be a full subcategory of $\mathscr{C}$. A weak colimit w. $\lim _{\rightarrow} A_{i}$ in $\mathscr{C}$ is called a $\mathscr{P}$-minimal weak colimit if the canonical morphism

$$
\lim _{\rightarrow} \mathscr{C}\left(\mathscr{P}, A_{i}\right) \rightarrow \mathscr{C}\left(\mathscr{P}, \mathrm{w} \cdot \lim _{\rightarrow} A_{i}\right)
$$

is an isomorphism. The subcategory $\mathscr{P}$ is called minimal if

(†) Every tower $A_{0} \rightarrow A_{1} \rightarrow A_{2} \rightarrow \cdots$ in $\mathscr{C}$ has a $\mathscr{P}$-minimal weak colimit.

Definition 3.3. A full subcategory $\mathscr{P}$ of an additive category $\mathscr{C}$ is called a Whitehead subcategory if $\mathscr{P}$ has the following properties.

(i) $\mathscr{P}$ is skeletally small.

(ii) A morphism $f: A \rightarrow B$ in $\mathscr{C}$ is an isomorphism iff $\mathscr{C}(\mathscr{P}, f)$ : $\mathscr{C}(\mathscr{P}, A) \rightarrow \mathscr{C}(\mathscr{P}, B)$ is an isomorphism.

COROLLARY 3.4. If the additive category $\mathscr{C}$ has countable coproducts and weak cokernels, then for any compact minimal Whitehead subcategory $\mathscr{P}$ of $\mathscr{C}$, any $\mathscr{P}$-minimal weak colimit of a tower is uniquely determined, by a not unique isomorphism.

Proof. Follows trivially from the fact that the functors $\mathscr{C}(P,-), P \in \mathscr{P}$, collectively reflect isomorphisms.

For latter use we state the following useful result.

LEMMA 3.5. Let $\mathscr{C}$ be an additive category with countable coproducts and weak cokernels, and let $\mathscr{X}$ be a covariantly finite subcategory of $\mathscr{C}$, satisfying the following:

(i) Any tower $A_{0} \stackrel{f_{0}}{\rightarrow} A_{1} \stackrel{f_{1}}{\rightarrow} A_{2} \rightarrow \cdots$ in $\mathscr{C}$, where each $f_{i}, i \in I$ is $\mathscr{X}$-monic, has a direct limit in $\mathscr{C}$.

(ii) If $\mathscr{P}$ is a full subcategory of $\mathscr{C}$ consisting of objects preserving the direct limits of (i), then $\mathscr{P}$ is closed under left $\mathscr{X}$-approximations.

Then $\mathscr{P} / \mathscr{X}$ is a minimal subcategory of $\mathscr{C} / \mathscr{X}$, i.e. any tower $\underline{A}_{0} \rightarrow \underline{A}_{1} \rightarrow$ $\underline{A}_{2} \rightarrow \cdots$ in $\mathscr{C} / \mathscr{X}$ has a $\mathscr{P} / \mathscr{X}$-minimal weak colimit.

PRoof. See Lemma 1.6 in [25]. 


\subsection{Brown Representability}

The concept of a Whitehead subcategory and of a $\mathscr{P}$-minimal weak colimit, enters in an essential way in the following Heller's version of the very important Brown Representability Theorem. We say that a contravariant, resp. covariant, functor $F: \mathscr{C} \rightarrow \mathscr{A} b$ is half-exact if for any diagram $A \stackrel{f}{\rightarrow} B \stackrel{g}{\rightarrow} C$ in $\mathscr{C}$, where $g$ is a weak cokernel of $f$, the sequence $F(C) \stackrel{F(g)}{\longrightarrow} F(B) \stackrel{F(f)}{\longrightarrow} F(A)$, resp. $F(A) \stackrel{F(f)}{\longrightarrow} F(B) \stackrel{F(g)}{\longrightarrow} F(C)$, is exact in $\mathscr{A} b$.

Theorem 3.6 (Brown). Suppose that $\mathscr{C}$ has coproducts and weak cokernels. If $\mathscr{C}$ contains a minimal Whitehead subcategory $\mathscr{P}$, then: an additive functor $H: \mathscr{C}^{o p} \rightarrow \mathscr{A} b$ is representable iff $H$ is half-exact and sends coproducts to products.

Proof. See [12], [20] for proofs in the non-additive setting.

Corollary 3.7. Under the assumptions of Theorem $3.6, \mathscr{C}$ has products and any object of $\mathscr{C}$ is in a unique way a weak colimit of a tower.

Proof. If $\left\{C_{i} ; i \in I\right\}$ is a set of objects of $\mathscr{C}$, then the functor $F: \mathscr{C}^{o p} \rightarrow$ $\mathscr{A} b$ defined by $F(A)=\prod \mathscr{C}\left(A, C_{i}\right)$ is half-exact and sends coproducts to products. Hence is representable with representing object the product of $\left\{C_{i} ; i \in I\right\}$. The proof of Brown Theorem in [20] applied to the functor $\mathscr{C}(-, A)$ shows that $A$ is a minimal weak colimit of a tower of objects.

TheOREM 3.8 (The Adjoint Theorem). Let $\mathscr{C}$ be an additive category with coproducts and weak cokernels and let $F: \mathscr{C} \rightarrow \mathscr{D}$ be an additive functor. If $\mathscr{C}$ admits a minimal Whitehead subcategory $\mathscr{P}$, then the following are equivalent:

(i) F has a right adjoint $G: \mathscr{D} \rightarrow \mathscr{C}$.

(ii) F preserves coproducts, weak cokernels.

In case $F$ has a right adjoint $G$, and the Whitehead subcategory $\mathscr{P}$ is compact, then the following are equivalent:

(iii) G preserves coproducts.

(iv) F preserves compact objects.

Proof. For the first part, it suffices to show that (ii) $\Rightarrow$ (i), since trivially any left adjoint preserves coproducts and weak cokernels. For any $D \in \mathscr{D}$, consider the functor $F_{D}: \mathscr{C}^{o p} \rightarrow \mathscr{A} b$ defined by $F_{D}(A)=\mathscr{D}(F(A), D)$. Obviously $F_{D}$ is half-exact and sends coproducts to products, so by Brown's Theorem, $F_{D}$ is representable. It is well known that this is equivalent to the existence of a right adjoint of $F$. Suppose that $F$ has a right adjoint $G$ and assume that $F$ preserves compact objects. Let $P \in \mathscr{P}$ and let $\left\{D_{i}, i \in I\right\}$ be a 
set of objects in $\mathscr{D}$. Then using that $P$ is compact and that $F$ preserves compact objects we have:

$$
\begin{gathered}
\mathscr{C}\left(P, G\left(\oplus D_{i}\right)\right) \cong \mathscr{D}\left(F(P), \oplus D_{i}\right) \cong \oplus \mathscr{D}\left(F(P), D_{i}\right) \cong \\
\cong \oplus \mathscr{C}\left(P, G\left(D_{i}\right)\right) \cong \mathscr{C}\left(P, \oplus G\left(D_{i}\right)\right) .
\end{gathered}
$$

Since this happens for any $P \in \mathscr{P}$ and $\mathscr{P}$ is a whitehead subcategory, we have $\oplus G\left(D_{i}\right) \cong G\left(\oplus D_{i}\right)$, so $G$ preserves coproducts. Conversely if $G$ preserves coproducts, let $X$ be a compact object in $\mathscr{C}$ and let $\left\{D_{i}, i \in I\right\}$ be a set of objects in $\mathscr{D}$. Then using adjointness and the fact that $G$ preserves coproducts, we have:

$$
\begin{gathered}
\mathscr{D}\left(F(X), \oplus D_{i}\right) \cong \mathscr{C}\left(X, G\left(\oplus D_{i}\right)\right) \cong \mathscr{C}\left(X, \oplus G\left(D_{i}\right)\right) \cong \\
\cong \oplus \mathscr{C}\left(X, G\left(D_{i}\right)\right) \cong \oplus \mathscr{D}\left(F(X), D_{i}\right) .
\end{gathered}
$$

Hence $F$ preserves compact objects.

DeFinition 3.9. Let $\mathscr{C}$ be an additive category with coproducts and weak cokernels. $\mathscr{C}$ is generated by a set $\mathscr{G} \subseteq \mathscr{C}$ if the smallest full additive subcategory of $\mathscr{C}$ which is closed under isomorphisms, coproducts and weak cokernels and contains $\mathscr{G}$, coincides with $\mathscr{C}$. Then $\mathscr{G}$ is called a generating set. The category $\mathscr{C}$ is called compactly generated if there exists a generating set $\mathscr{G}$ consisting of compact objects.

COROLlary 3.10. Let $\mathscr{C}$ be an additive category with coproducts and weak cokernels and let $\mathscr{P}$ be a minimal Whitehead subcategory of $\mathscr{C}$. Then $\mathscr{C}$ is generated by $\operatorname{Iso}(\mathscr{P})$. Hence if the Whitehead subcategory $\mathscr{P}$ is compact, then $\mathscr{C}$ is compactly generated.

Proof. Let $\mathscr{U}$ be the smallest full additive subcategory of $\mathscr{C}$ which is closed under isomorphisms, coproducts and weak cokernels and contains $\mathscr{P}$. Then $\mathscr{U}$ has coproducts and weak cokernels and $\mathscr{P}$ is a minimal Whitehead subcategory of $\mathscr{U}$. Let $A$ be an object in $\mathscr{C}$ and consider the functor $F=$ $\left.\mathscr{C}(-, A)\right|_{\mathscr{U}}: \mathscr{U}^{o p} \rightarrow \mathscr{A} b$. Since $F$ sends coproducts to products and is half-

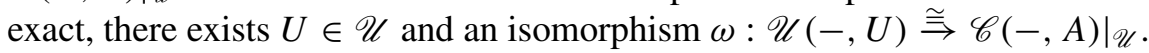
Then the morphism $\omega\left(1_{U}\right): U \rightarrow A$, has the property that $\mathscr{C}\left(\mathscr{P}, \omega\left(1_{U}\right)\right)$ is an isomorphism. Since $\mathscr{P}$ is Whitehead, $\omega\left(1_{U}\right)$ is an isomorphism. Hence $A \in \mathscr{U}$. We conclude that $\mathscr{C}=\mathscr{U}$.

\subsection{Right Triangulated Categories}

We recall from [9], [27], that a left, resp. right, triangulated category is an additive category $\mathscr{C}$ equipped with an additive endofunctor $\Omega: \mathscr{C} \rightarrow \mathscr{C}$ the 
loop functor, resp. $\Sigma: \mathscr{C} \rightarrow \mathscr{C}$ the suspension functor, and a class of diagrams $\Delta$, resp. $\nabla$, of the form $\Omega(C) \rightarrow A \rightarrow B \rightarrow C$, resp. of the form $A \rightarrow$ $B \rightarrow C \rightarrow \Sigma(A)$, called triangles, satisfying all the axioms of a triangulated category [37], except that $\Omega$, resp. $\Sigma$, is not necessarily an equivalence. If $\mathscr{C}$ is a left or right triangulated category, then (contravariant) half exact functors are called (cohomological) homological functors: they send triangles to exact sequences. If $\mathscr{C}$ is left triangulated then $\mathscr{C}(A,-)$ is homological and if the loop functor $\Omega$ is fully faithful then $\mathscr{C}(-, A)$ is cohomological. If $\mathscr{C}$ is right triangulated then $\mathscr{C}(-, A)$ is cohomological and if the suspension functor $\Sigma$ is fully faithful then $\mathscr{C}(A,-)$ is homological.

We recall that if $\mathscr{C}$ is a (left or right) triangulated category, then a subcategory $\mathscr{E}$ of $\mathscr{C}$ is called thick if $\mathscr{E}$ is a full additive (left or right) triangulated subcategory of $\mathscr{C}$, which is closed under direct summands. If $\mathscr{C}$ has coproducts, then a thick subcategory $\mathscr{L}$ of $\mathscr{C}$ is called localizing if $\mathscr{L}$ is closed under coproducts. A (left or right) triangulated category $\mathscr{C}$ is called compactly generated if $\mathscr{C}$ has coproducts and a set $\mathscr{S}$ of compact objects, such that $\mathscr{C}$ coincides with the smallest thick subcategory of $\mathscr{C}$ which contains $\mathscr{S}$ and is closed under isomorphisms and coproducts.

Let $\mathscr{C}$ be a right triangulated category with coproducts. We define a full subcategory $\widehat{\mathscr{C}^{b}}$ of $\mathscr{C}$ as follows:

$$
\widehat{\mathscr{C}^{b}}:=\left\{X \in \mathscr{C}^{b} \mid \text { the functor } \mathscr{C}(X,-): \mathscr{C} \rightarrow \mathscr{A} b \text { is homological }\right\} .
$$

Hence a compact object $X$ is in $\widehat{\mathscr{C}^{b}}$ iff for any triangle $A \stackrel{f}{\rightarrow} B \stackrel{g}{\rightarrow} C \stackrel{h}{\rightarrow} \Sigma(A)$ in $\mathscr{C}$, any morphism $\alpha: X \rightarrow B$ such that $\alpha \circ g=0$ factors through $f$. Hence if $\Sigma$ is fully faithful, in particular if $\mathscr{C}$ is triangulated, then $\widehat{\mathscr{C}^{b}}=\mathscr{C}^{b}$.

LEMMA 3.11. If the suspension functor $\Sigma$ preserves countable coproducts, then any full subcategory $\mathscr{P}$ of $\widehat{\mathscr{C}}^{b}$ is minimal. Hence if $\mathscr{C}$ contains a Whitehead subcategory $\mathscr{P} \subseteq \mathscr{C}^{b}$, then $\mathscr{C}$ is compactly generated.

Proof. Let $A_{1} \rightarrow A_{2} \rightarrow \cdots$ be a tower of objects of $\mathscr{C}$, and consider the induced triangle $\oplus_{i \geq 1} A_{i} \rightarrow \oplus_{i \geq 1} A_{i} \rightarrow$ w. $\lim A_{i} \rightarrow \Sigma\left(\oplus_{i \geq 1} A_{i}\right)$. If $X \in$ $\mathscr{P}$, then applying the half-exact functor $\mathscr{C}(X, \overrightarrow{-})$ to the above triangle and using that $X$ is compact and $\Sigma$ preserves countable coproducts, we conclude directly that the canonical morphism $\lim _{\rightarrow} \mathscr{C}\left(X, A_{i}\right) \rightarrow \mathscr{C}\left(X\right.$, w. $\left.\lim _{\rightarrow} A_{i}\right)$ is an isomorphism, so $\mathscr{P}$ is minimal. It follows that if moreover $\mathscr{P}$ is Whitehead, then $\mathscr{C}$ is compactly generated by $\mathscr{P}$.

COROLlaRY 3.12. Let $\mathscr{C}$ be a right triangulated category with coproducts and suspension functor $\Sigma$. If $\mathscr{C}$ contains a compact minimal Whitehead subcategory, then: 
(i) $\mathscr{C}$ is compactly generated with products.

(ii) $F: \mathscr{C}^{o p} \rightarrow \mathscr{A} b$ is representable $\Leftrightarrow F$ is cohomological and sends coproducts to products.

(iii) The suspension functor $\Sigma$ has a right adjoint $\Omega \Leftrightarrow \Sigma$ preserves coproducts. If $\Omega$ exists then $\Omega$ preserves coproducts $\Leftrightarrow \Sigma$ preserves compact objects.

If $\mathscr{L}$ is a thick subcategory of the right triangulated category $\mathscr{C}$, then we can define the quotient category $\mathscr{C} / \mathscr{L}$, in a possibly larger universe, as in the triangulated case by formally inverting [17] all morphisms $A \rightarrow B$ in $\mathscr{C}$, such that in the triangle $A \rightarrow B \rightarrow L \rightarrow \Sigma(A)$ the object $L \in \mathscr{L}$. Then $\mathscr{C} / \mathscr{L}$ is a right triangulated category and the quotient functor $q: \mathscr{C} \rightarrow \mathscr{C} / \mathscr{L}$ is exact. If $\mathscr{C}$ has coproducts and $\mathscr{L}$ is localizing, then $\mathscr{C} / \mathscr{L}$ has coproducts and the functor $q$ preserves them. The next result which follows from Theorem 3.8 and Lemma 3.11, is a version of Bousfield's localization in right triangulated categories.

Proposition 3.13. Let $\mathscr{C}$ be a right triangulated category with coproducts and let $\mathscr{L}$ be a localizing subcategory of $\mathscr{C}$.

(i) If $\mathscr{C}$ is compactly generated and the quotient category $\mathscr{C} / \mathscr{L}$ has small hom-sets, then the quotient functor $q: \mathscr{C} \rightarrow \mathscr{C} / \mathscr{L}$ has a right adjoint.

(ii) If $\mathscr{L}$ is generated by a Whitehead subcategory $\mathscr{P}$ of $\mathscr{C}$ contained in $\widehat{\mathscr{C}^{b}}$, then $\mathscr{L}$ is compactly generated and the inclusion functor $i: \mathscr{L} \hookrightarrow \mathscr{C}$ has a right adjoint.

\section{4. $t$-Structures}

We recall that if $\mathscr{U}$ is a full subcategory of $\mathscr{C}$ then $\mathscr{U}^{\perp}$ denotes the full subcategory $\{A \in \mathscr{C} \mid \mathscr{C}(U, A)=0, \forall U \in \mathscr{U}\}$. For the concept of a $t$-structure in a triangulated category we refer to [4].

PROPOSITION 3.14. Let $\mathscr{C}$ be a triangulated category with coproducts and suspension functor $\Sigma$. Let $\mathscr{U}$ be a full additive subcategory of $\mathscr{C}$ closed under extensions, coproducts and the suspension functor $\Sigma$.

(1) If there exists a Whitehead subcategory $\mathscr{P}$ of $\mathscr{U}$ contained in $\mathscr{U}^{b}$, then the pair $\left(\mathscr{U}, \Sigma \mathscr{U}^{\perp}\right)$ is a $t$-structure in $\mathscr{C}$.

(2) If $\mathscr{C}$ is compactly generated and the quotient $\mathscr{C} / \mathscr{U}$ has small hom-sets, then $\left(\mathscr{U}, \Sigma \mathscr{U}^{\perp}\right)$ is a $t$-structure in $\mathscr{C}$.

Proof. The hypothesis implies that $\mathscr{U}$ is a right triangulated subcategory of $\mathscr{C}$. If (1) holds, then by Theorem 3.8 and Lemma 3.11 it follows that the inclusion $\mathscr{U} \hookrightarrow \mathscr{C}$ has a right adjoint. Then the result follows from [28]. 
Suppose that condition (2) holds. Since $\mathscr{C} / \mathscr{U}$ has small hom-sets and $\mathscr{C}$ is compactly generated, by Theorem 3.8 the quotient functor $q: \mathscr{C} \rightarrow \mathscr{C} / \mathscr{U}$ has a right adjoint $r$. Let $\delta: \operatorname{Id}_{\mathscr{C}} \rightarrow r q$ be the unit of the adjoint pair $(q, r)$. Then for any object $A$ in $\mathscr{C}$ we have a triangle $A^{\prime} \rightarrow A \stackrel{\delta_{A}}{\rightarrow} r q(A) \rightarrow \Sigma\left(A^{\prime}\right)$ in $\mathscr{C}$. Since obviously $q\left(A^{\prime}\right)=0$, it follows that $A^{\prime} \in \mathscr{U}$. For any $U \in \mathscr{U}$ we have the long exact sequence

$$
\cdots \rightarrow \mathscr{C}\left(U, \Sigma^{-1} r q(A)\right) \rightarrow \mathscr{C}\left(U, A^{\prime}\right) \rightarrow \mathscr{C}(U, A) \rightarrow \mathscr{C}(U, r q(A)) \rightarrow \cdots
$$

Then $\mathscr{C}\left(U, \Sigma^{-1} r q(A)\right) \cong \mathscr{C}(\Sigma(U), r q(A)) \cong \mathscr{C} / \mathscr{U}(q \Sigma(U), q(A))=0$. In the same way $\mathscr{C}(U, r q(A))=0$. Hence $\mathscr{C}\left(U, A^{\prime}\right) \cong \mathscr{C}(U, A)$. This shows that $A^{\prime}$ is the coreflection of $A$ in $\mathscr{U}$. Hence the inclusion $\mathscr{U} \hookrightarrow \mathscr{C}$ has a right adjoint and then by [28], the pair $\left(\mathscr{U}, \Sigma \mathscr{U}^{\perp}\right)$ is a $t$-structure in $\mathscr{C}$.

Let $\mathscr{C}$ be a compactly generated triangulated category and let $\mathbf{T}$ be the class of $t$-structures in $\mathscr{C}$. Also let $\mathbf{U}$ be the class of right triangulated subcategories of $\mathscr{C}$ which are closed under coproducts and such that the quotient $\mathscr{C} / \mathscr{U}$ has small hom-sets, for any $\mathscr{U} \in \mathbf{U}$. By the above result we have the following.

Corollary 3.15. The assignement $\mathscr{U} \longmapsto\left(\mathscr{U}, \Sigma \mathscr{U}^{\perp}\right)$ gives a bijective correspondence between the classes $\mathbf{U}$ and $\mathbf{T}$.

Example 3.16. Let $\mathscr{D}(\Lambda)$ be the unbounded derived category of right modules over a ring $\Lambda$. It is well known that $\mathscr{D}(\Lambda)$ is a compactly generated triangulated category. Let $\mathscr{U}$ be the right triangulated subcategory of $\mathscr{D}(\Lambda)$ generated by $\Lambda$, i.e. the smallest right triangulated subcategory of $\mathscr{D}(\Lambda)$ which contains $\Lambda$ and is closed under coproducts. Then the $t$-structure of the above proposition is the natural $t$-structure in $\mathscr{D}(\Lambda)$, see [4].

\subsection{Triangulated Categories}

Corollary 3.17. If $\mathscr{C}$ is triangulated, then the following are equivalent:

(i) $\mathscr{C}$ is compactly generated.

(ii) $\mathscr{C}$ has coproducts and there exists a set of compact objects $\mathscr{S}$, such that $\Sigma(\mathscr{S})=\mathscr{S}$ and $\mathscr{C}(\mathscr{S}, A)=0 \Rightarrow A=0$.

(iii) $\mathscr{C}$ has coproducts and contains a full compact Whitehead subcategory.

(iv) $\mathscr{C}$ has coproducts, $\mathscr{C}^{b}$ is skeletally small and $\mathscr{C}\left(\mathscr{C}^{b}, A\right)=0 \Rightarrow A=0$.

Proof. (i) $\Rightarrow$ (ii) If $\mathscr{S}$ is a generating set, then $\bigcup_{n \in Z} \Sigma^{n}(\mathscr{S})$ is also a generating set which is closed under suspension, hence we can assume that $\Sigma(\mathscr{S})=\mathscr{S}$. Let $\mathscr{C}(\mathscr{S}, A)=0$ and consider the full subcategory $\mathscr{U}_{A}:=\{X \in$ $\left.\mathscr{C} ; \forall n \in Z: \mathscr{C}\left(X, \Sigma^{n}(A)\right)=0\right\}$. Then $\mathscr{U}_{A}$ is a triangulated subcategory of $\mathscr{C}$, 
closed under coproducts, isomorphisms, and contains $\mathscr{S}$. Hence $\mathscr{U}_{A}=\mathscr{C}$ and then obviously $A=0$.

(ii) $\Rightarrow$ (iii) Let $f: A \rightarrow B$ be a morphism in $\mathscr{C}$ with $\mathscr{C}(\mathscr{S}, f)$ an isomorphism. If $A \stackrel{f}{\rightarrow} B \rightarrow C \rightarrow \Sigma(A)$ is a triangle in $\mathscr{C}$, then since $\Sigma(\mathscr{S})=$ $\mathscr{S}$, it follows that $\mathscr{C}(\mathscr{S}, C)=0$, hence by hypothesis $C=0$. Then trivially $f$ is an isomorphism.

(iii) $\Rightarrow$ (i) It is easy to see that any compact subcategory of $\mathscr{C}$ which is closed under suspension, is minimal. Then the assertion follows from Corollary 3.12 .

(iv) $\Leftrightarrow$ (ii) Obviously (iv) implies (ii). Suppose that (ii) is true. It suffices to show that $\mathscr{C}^{b}$ is skeletally small. Let $\mathscr{S}$ be the thick subcategory generated by $\mathscr{S}$. Then obviously $\widehat{\mathscr{S}}$ is skeletally small and $\widehat{\mathscr{S}} \subseteq \mathscr{C}^{b}$. By [26], we have that any compact object of $\mathscr{C}$ is a direct summand of an extension of objects of $\mathscr{S}$. This trivially implies that $\widehat{\mathscr{S}}=\mathscr{C}^{b}$, hence $\mathscr{C}^{b}$ is skeletally small.

If $\mathscr{C}$ is triangulated and $\mathscr{L}$ is a thick subcategory of $\mathscr{C}$, then by [37] we have that the inclusion $\mathscr{L} \hookrightarrow \mathscr{C}$ has a right adjoint iff the quotient functor $\mathscr{C} \rightarrow \mathscr{C} / \mathscr{L}$ has a right adjoint, provided that $\mathscr{C} / \mathscr{L}$ has small hom-sets. In this case $\mathscr{C} / \mathscr{L}=\mathscr{L}^{\perp}$. Hence by the above results, we have the following Corollary which is the fundamental result in the theory of compactly generated triangulated categories.

CoROllary 3.18 ([32], [33], [26]). Let $\mathscr{C}$ be a compactly generated triangulated category.

(1) An additive functor $H: \mathscr{C}^{o p} \rightarrow \mathscr{A} b$ is representable iff $H$ is cohomological and sends coproducts to products. In particular $\mathscr{C}$ has products.

(2) An exact functor $F: \mathscr{C} \rightarrow \mathscr{D}$ to the triangulated category $\mathscr{D}$, has a right adjoint iff $F$ preserves coproducts. In case the right adjoint $G$ of $F$ exists, then $G$ has a right adjoint iff $F$ preserves compact objects.

(3) If $\mathscr{L}$ is a localizing subcategory of $\mathscr{C}$, then: the inclusion functor $\mathscr{L} \hookrightarrow$ $\mathscr{C}$ has a right adjoint iff the quotient functor $\mathscr{C} \rightarrow \mathscr{C} / \mathscr{L}$ has a right adjoint iff $\mathscr{C} / \mathscr{L}$ has small hom-sets iff the canonical functor $\mathscr{L}^{\perp} \rightarrow \mathscr{C} \mid \mathscr{L}$ is an equivalence.

\subsection{Locally Finitely Presented Additive Categories}

Brown Representability has also interesting applications to locally finiteley presented categories. We have the following consequences of Theorems 3.6, 3.8 .

THEOREM 3.19. Let $\mathscr{C}$ be a locally finitely presented additive category. Then the following are equivalent:

(i) $\mathscr{C}$ has products. 
(ii) $\mathscr{C}$ is covariantly finite in $\operatorname{Mod}(\mathrm{f} . \mathrm{p} .(\mathscr{C}))$.

(iii) $\mathscr{C}$ has weak cokernels.

(iv) An additive functor $H: \mathscr{C}^{o p} \rightarrow \mathscr{A} b$ is representable iff $H$ is half-exact and sends coproducts to products.

Proof. By [13], $\mathscr{C}$ is equivalent to the category Flat $(\operatorname{Mod}(f . p .(\mathscr{C})))$ of flat contravariant additive functors from its subcategory f.p. $(\mathscr{C})$ of finitely presented objects to $\mathscr{A} b$. If $\mathscr{C}$ has products, then by [13] the subcategory f.p. $(\mathscr{C})$ has weak cokernels. Then by [5], Flat $(\operatorname{Mod}(f . p .(\mathscr{C})))$ is covariantly finite in $\operatorname{Mod}($ f.p. $(\mathscr{C}))$. If $\mathscr{C}$ is covariantly finite in $\operatorname{Mod}(f . p .(\mathscr{C}))$, then by Proposition 3.1, $\mathscr{C}$ has weak cokernels. If $\mathscr{C}$ has weak cokernels then since $\mathscr{C}$ has coproducts and f.p. $(\mathscr{C})$ is a compact minimal Whitehead subcategory of $\mathscr{C}$, the assertion (iv) follows from Brown's Theorem. If Brown's Theorem holds in $\mathscr{C}$ then as in Corollary 3.7, $\mathscr{C}$ has products.

THEOREM 3.20 ([30]). Let $\mathscr{C}, \mathscr{D}$ be locally finitely presented additive categories with products and let $F: \mathscr{C} \rightarrow \mathscr{D}$ be an additive functor. The following are equivalent:

(i) F has a right adjoint $G: \mathscr{D} \rightarrow \mathscr{C}$ (and $G$ preserves coproducts, resp. direct limits).

(ii) $F$ preserves coproducts, weak cokernels (and compact, resp. finitely presented objects).

PROOF. If $\mathscr{C}$ is a locally finitely presented category with products, then by the above Theorem, f.p. $(\mathscr{C})$ is a compact Whitehead subcategory of $\mathscr{C}$ and the assertions are consequences of Brown's Theorem. We include a proof of the parenthetical cases. By the Adjoint Theorem, the right adjoint $G$ of $F$ preserves coproducts iff $F$ preserves compact objects. Assume that $G$ preserves direct limits and let $X \in$ f.p.( $\mathscr{C})$. Then $\mathscr{D}\left(F(X), \lim _{\rightarrow} D_{i}\right) \cong \mathscr{C}\left(X, G\left(\lim _{\rightarrow} D_{i}\right)\right) \cong$ $\mathscr{C}\left(X, \lim _{\rightarrow} G\left(D_{i}\right)\right) \cong \lim _{\rightarrow} \mathscr{C}\left(X, G\left(D_{i}\right)\right) \cong \lim _{\rightarrow} \mathscr{D}\left(F(X), D_{i}\right)$. Hence $F(X)$ is finitely presented. Conversely if $F$ preserves coproducts, weak cokernels and finitely presented objects, then $\forall X \in$ f.p. $(\mathscr{C})$ we have:

$$
\begin{gathered}
\mathscr{C}\left(X, G\left(\lim _{\rightarrow} D_{i}\right)\right) \cong \mathscr{D}\left(F(X), \lim _{\rightarrow} D_{i}\right) \cong \lim _{\rightarrow} \mathscr{D}\left(F(X), D_{i}\right) \\
\cong \lim _{\rightarrow} \mathscr{C}\left(X, G\left(D_{i}\right)\right) \cong \mathscr{C}\left(X, \lim _{\rightarrow} G\left(D_{i}\right)\right) .
\end{gathered}
$$

Since f.p.(C) is Whitehead, it follows that $\lim _{\rightarrow} G\left(D_{i}\right) \cong G\left(\lim _{\rightarrow} D_{i}\right)$, hence $G$ preserves direct limits. 


\subsection{Cohomology Theories and Costabilization}

Let $\mathscr{C}$ be a right triangulated category with (right) triangulation $\nabla$ and suspension functor $\Sigma$. A cohomology theory on $\mathscr{C}$ is a sequence $\left\{H^{n} ; h^{n}\right\}_{n \in \mathrm{Z}}$, where $H^{n}: \mathscr{C}^{o p} \rightarrow \mathscr{A} b$ is a cohomological functor and $h^{n}: H^{n} \stackrel{\cong}{\rightrightarrows} H^{n+1} \Sigma$ are natural isomorphisms. A morphism between the cohomology theories $\left\{H^{n} ; h^{n}\right\}_{n \in \mathrm{Z}},\left\{F^{n} ; f^{n}\right\}_{n \in \mathrm{Z}}$ is a sequence of morphisms $\left\{\alpha^{n}: H^{n} \rightarrow F^{n}\right\}_{n \in \mathrm{Z}}$, such that $\forall n \in Z: \alpha^{n} \circ f^{n}=h^{n} \circ \alpha^{n+1} \Sigma$. We denote by $\operatorname{Coh} T h(\mathscr{C})$ the category of cohomology theories on $\mathscr{C}$. If $\mathscr{C}$ has coproducts, then we denote by $\widehat{\operatorname{CohTh}}(\mathscr{C}, \Sigma, \nabla)$ the full subcategory consisting of all cohomology theories $\left\{H^{n} ; h^{n}\right\}_{n \in Z}$ such that $H^{n}$ sends coproducts to products, $\forall n \in Z$. Our aim in this subsection is to describe in some cases the category $\widehat{\operatorname{CohTh}}(\mathscr{C}, \Sigma, \nabla)$. To this end we need some definitions.

Suppose that $\mathscr{C}, \mathscr{D}$ are additive categories equipped with endofuctors $\Omega, \Sigma$ respectively. A functor $F: \mathscr{C} \rightarrow \mathscr{D}$ is called stable if $F \Omega=\Sigma F$. If $\Omega: \mathscr{C} \rightarrow \mathscr{C}$ is an endofunctor of an additive category $\mathscr{C}$, then by [8], there exists a couniversal category in which $\Omega$ becomes invertible in the following sense. There exists a pair $(\mathscr{R}(\mathscr{C}, \Omega), \mathbf{R})$ consisting of a category $\mathscr{R}(\mathscr{C}, \Omega)$ equipped with an equivalence $\widehat{\Omega}: \mathscr{R}(\mathscr{C}, \Omega) \approx \mathscr{\rightarrow} \mathscr{R}(\mathscr{C}, \Omega)$ and a stable functor $\mathbf{R}: \mathscr{R}(\mathscr{C}, \Omega) \rightarrow \mathscr{C}$, such that for any stable functor $F: \mathscr{D} \rightarrow \mathscr{C}$ from a category $\mathscr{D}$ equipped with an autoequivalence, there exists a unique (up to isomorphism) stable functor $F^{*}: \mathscr{D} \rightarrow \mathscr{R}(\mathscr{C}, \Omega)$ such that $\mathbf{R} F^{*}=F$. The category $\mathscr{R}(\mathscr{C}, \Omega)$ is called the costabilization of $\mathscr{C}$ (with respect to $\Omega$ ) and the stable functor $\mathbf{R}$ is called the costabilization functor. If the pair $(\mathscr{C}, \Omega)$ admits a left or right triangulation $\Delta$, then the costabilization of $\mathscr{C}$ is triangulated, and is denoted by $\mathscr{R}(\mathscr{C}, \Omega, \Delta)$. In this case the costabilization functor $\mathbf{R}$ is exact and the pair is couniversal for exact functors from triangulated categories to $\mathscr{C}$. We recall the description of $\mathscr{R}(\mathscr{C}, \Omega)$ from [8]. The objects of $\mathscr{R}(\mathscr{C}, \Omega)$ are sequences $\left\{A_{n}, \alpha_{n}\right\}_{n \in Z}$, where $\alpha_{n}: A_{n} \cong \mathscr{C} \cong\left(A_{n+1}\right)$ are isomorphisms in $\mathscr{C}$. A morphism between the objects $\left\{A_{n}, \alpha_{n}\right\}_{n \in \mathrm{Z}},\left\{B_{n}, \beta_{n}\right\}_{n \in \mathrm{Z}}$ is a sequence of morphisms $\chi_{n}: A_{n} \rightarrow B_{n}$, such that $\alpha_{n} \circ \Omega\left(\chi_{n+1}\right)=\chi_{n} \circ \beta_{n}, \forall n \in Z$. The costabilization functor is defined by setting $\mathbf{R}\left(\left\{A_{n}, \alpha_{n}\right\}_{n \in Z}\right)=A_{0}$.

The following generalizes a result of Jørgensen [25].

TheOREM 3.21. Let $(\mathscr{C}, \Sigma, \nabla)$ be a right triangulated category with coproducts containing a minimal Whitehead subcategory and let $\Omega$ be a right adjoint of $\Sigma$.

Then there exists a full embedding $\mathrm{T}: \mathscr{R}(\mathscr{C}, \Omega) \hookrightarrow \operatorname{Coh} \mathrm{T}(\mathscr{C}, \Sigma, \nabla)$ which induces an equivalence $\mathrm{T}: \mathscr{R}(\mathscr{C}, \Omega) \approx \widehat{\operatorname{CohTh}}(\mathscr{C}, \Sigma, \nabla)$. If $(\mathscr{C}, \Omega)$ admits a left triangulation $\Delta$, then $\widehat{\operatorname{CohT}} h(\mathscr{C}, \Sigma, \nabla) \approx \mathscr{R}(\mathscr{C}, \Omega, \Delta)$ is triangulated with coproducts. 
Proof. Let $\phi_{-, ?}: \mathscr{C}(\Sigma(-)$, ?) $\cong \mathscr{C}(-, \Omega(?))$ be the natural isomorphism associated to the adjoint pair $(\Sigma, \Omega)$. Define a functor $\mathrm{T}: \mathscr{R}(\mathscr{C}, \Omega) \hookrightarrow$ $\operatorname{CohT} h(\mathscr{C}, \Sigma, \nabla)$ as follows: $\mathrm{T}\left(\left\{A_{n} ; \alpha_{n}\right\}_{n \in Z}\right)=\left\{H^{n} ; h^{n}\right\}_{n \in Z}$, where $H^{n}:=$ $\mathscr{C}\left(-, A_{n}\right)$ and $h^{n}:=\mathscr{C}\left(-, \alpha_{n}\right) \circ \phi_{-, A_{n+1}}: \mathscr{C}\left(-, A_{n}\right) \rightarrow \mathscr{C}\left(\Sigma(-), A_{n+1}\right)$. If $\left(\chi_{n}\right)_{n \in Z}:\left\{A_{n} ; \alpha_{n}\right\}_{n \in Z} \rightarrow\left\{A_{n} ; \alpha_{n}\right\}_{n \in Z}$ is a morphism in $\mathscr{R}(\mathscr{C}, \Omega)$, then $\mathrm{T}\left(\left(\chi_{n}\right)_{n \in \mathrm{Z}}\right):=\left\{\mathscr{C}\left(-, \chi_{n}\right)\right\}_{n \in \mathrm{Z}}$. Trivially $\mathrm{T}$ is a full embedding. If $\left\{H^{n} ; h^{n}\right\}_{n \in \mathrm{Z}}$ is a cohomology theory, where each $H^{n}$ sends coproducts to products, then by Brown Representability we have natural isomorphisms $\tau_{n}: H^{n} \cong \mathscr{C}\left(-, A_{n}\right)$ , $\forall n \in$ Z. Define isomorphisms $\alpha_{n}: A_{n} \rightarrow \Omega\left(A_{n+1}\right)$ by the composition of isomorphisms $\alpha_{n}:=\tau_{n} \circ h^{n} \circ \tau_{n+1} \Sigma \circ \phi_{-, A_{n+1}}$. Then $\left\{A_{n} ; \alpha_{n}\right\}_{n \in \mathrm{Z}}$ is in $\mathscr{R}(\mathscr{C}, \Omega)$ and $\mathbf{R}\left(\left\{A_{n} ; \alpha_{n}\right\}_{n \in \mathrm{Z}}\right)=\left\{H^{n} ; h^{n}\right\}_{n \in \mathrm{Z}}$.

COROLlARY 3.22. Let $\mathscr{C}$ be a compactly generated triangulated category. Then there exists a triangle equivalence $\mathscr{C} \approx \widehat{\mathrm{CohTh}}(\mathscr{C})$.

\section{Homotopy Pairs and Closed Model Categories}

\subsection{Closed Model Categories and Functorially Finite Subcategories}

Let $\mathscr{C}$ be an additive category. We recall the concept of a (closed) model structure on $\mathscr{C}$ in the sense of Quillen [35], [14], [22].

Definition 4.1. A (closed) model structure on $\mathscr{C}$ consists of three classes of morphisms of $\mathscr{C}$ :

(1) $\operatorname{Cof}(\mathscr{C})$, the class of cofibrations,

(2) $F i b(\mathscr{C})$, the class of fibrations,

(3) Weq $(\mathscr{C})$, the class of weak equivalences,

satisfying the following properties.

(i) If $f, g$ are morphisms in $\mathscr{C}$ such that the composition $f \circ g$ is defined and two of $f, g, f \circ g$ are weak equivalences, then so is the third.

(ii) If $f$ is a retract of $g$ in the category $\mathscr{C}^{2}$ of morphisms of $\mathscr{C}$ and $g$ is a cofribation, fibration or weak equivalence, then so is $f$.

(iii) Define a morphism $f$ to be a trivial fibration if $f$ is both a fibration and a weak equivalence. Define a morphism $f$ to be a trivial cofibration if $f$ is both a cofibration and a weak equivalence. Then for any commutative diagram

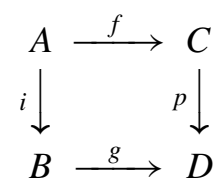


where $i$ is a trivial cofibration and $p$ is a fibration or $i$ is a cofibration and $p$ is a trivial fibration, there exists $h: B \rightarrow C$ such that $i \circ h=f$ and $h \circ p=g$.

(iv) For any morphism $f$ in $\mathscr{C}$ there are factorizations: $f=f_{1} \circ f_{2}$ and $f=f_{3} \circ f_{4}$ in $\mathscr{C}$ such that: $f_{1}$ is a cofibration, $f_{2}$ is a trivial fibration, $f_{3}$ is a trivial cofibration and $f_{4}$ is a fibration.

If $\mathscr{C}$ admits a closed model structure, then an object $A \in \mathscr{C}$ is called fibrant, resp. cofibrant, if the morphism $A \rightarrow 0$ is a fibration, resp. $0 \rightarrow A$ is a cofibration. An object $A$ is called bifibrant if $A$ it is fibrant and cofibrant.

DeFINITION 4.2. The homotopy category $\operatorname{Ho}(\mathscr{C})$ of $\mathscr{C}$ with respect to a closed model structure $(\operatorname{Cof}(\mathscr{C}), \mathrm{Fib}(\mathscr{C})$, Weq $(\mathscr{C}))$, is defined to be the category $\operatorname{Ho}(\mathscr{C}):=\mathscr{C}\left[W e q(\mathscr{C})^{-1}\right]$ obtained by formally inverting the class of weak equivalences, see [17].

Quillen [35] defines (in the additive case) a closed model category as an additive category $\mathscr{C}$ with kernels and cokernels, together with a closed model structure on $\mathscr{C}$. For our purposes, we need a weaker notion.

DEFINITION 4.3. A weak closed model category is an additive category $\mathscr{C}$ together with a closed model structure $(\operatorname{Cof}(\mathscr{C}), F i b(\mathscr{C})$, Weq $(\mathscr{C}))$ on $\mathscr{C}$, such that any cofibration has a cokernel and any fibration has a kernel.

We fix in this section an additive category $\mathscr{C}$ with split idempotents. Let $\mathscr{X} \subseteq \mathscr{C}$ be a full additive subcategory of $\mathscr{C}$, closed under direct summands and isomorphisms. Define classes of morphisms in $\mathscr{C}$ as follows:

(i) $\operatorname{Cof}_{\mathscr{X}}(\mathscr{C})$ is the class of $\mathscr{X}$-monics.

(ii) $F i b_{\mathscr{X}}(\mathscr{C})$ is the class of $\mathscr{X}$-epics.

(iii) $W e q_{\mathscr{X}}(\mathscr{C})$ is the class of stable equivalences, i.e. $f \in W e q(\mathscr{C})$ iff $f$ is an isomorphism in $\mathscr{C} / \mathscr{X}$.

Let $f, g: A \rightarrow B$ be two morphisms in $\mathscr{C}$. We say that $f, g$ are $\mathscr{X}$ homotopic, if $f-g$ factors through an object of $\mathscr{X}$, i.e. if $\underline{f}=g$ in the stable category $\mathscr{C} / \mathscr{X}$.

Lemma 4.4. Let $f: A \rightarrow B$ be a morphism in $\mathscr{C}$.

(i) $f$ is a trivial cofibration iff $f$ is split monic and Coker $(f) \in \mathscr{X}$.

(ii) $f$ is a trivial fibration iff $f$ is split epic and $\operatorname{Ker}(f) \in \mathscr{X}$.

Proof. If $f$ is a trivial cofibration then $f$ is an isomorphism in $\mathscr{C} / \mathscr{X}$, so there is a morphism $\underline{g}: \underline{B} \rightarrow \underline{A}$ such that $\overline{1}_{\underline{A}}=\underline{f} \circ \underline{g}$. Then the morphism $1_{A}-f \circ g$ factors through an object of $\mathscr{X}$; hence there are morphisms $\chi$ : $A \rightarrow X, \kappa: X \rightarrow A$, where $X \in \mathscr{X}$, such that: $1_{A}-f \circ g=\chi \circ \kappa$. Since 
$f$ is $\mathscr{X}$-monic, there exists a morphism $\lambda: B \rightarrow X$ such that $\chi=f \circ \lambda$. Then $1_{A}-f \circ g=f \circ \lambda \circ \kappa \Rightarrow 1_{A}=f \circ(g+\lambda \circ \kappa)$, so $f$ is split monic. Since idempotents split in $\mathscr{C}, f$ has a cokernel and then $\operatorname{Coker}(f) \in \mathscr{X}$. Conversely if $f$ is split monic then trivially $f$ is a cofibration. Since $f$ induces an isomorphism $B=A \oplus \operatorname{Coker}(f)$ in $\mathscr{C}$, if in addition $\operatorname{Coker}(f) \in \mathscr{X}$, then $f$ is an isomorphism in $\mathscr{C} / \mathscr{X}$. The proof of (2) is dual.

Our main result of this section is the following.

THEOREM 4.5. The following are equivalent:

(i) $\mathscr{X}$ is a functorially finite subcategory of $\mathscr{C}$.

(ii) The triple $\left(\operatorname{Cof}_{\mathscr{X}}(\mathscr{C})\right.$, Fib $_{\mathscr{X}}(\mathscr{C})$, Weq $\left.q_{\mathscr{X}}(\mathscr{C})\right)$ is a closed model structure on $\mathscr{C}$.

In this case all objects of $\mathscr{C}$ are bifibrant and the associated homotopy category $\mathscr{C}\left[W e q_{\mathscr{X}}(\mathscr{C})^{-1}\right]$ is equivalent to the stable category $\mathscr{C} / \mathscr{X}$. Finally the left or right homotopy relation (see [35]) induced from the closed model structure of $\mathscr{C}$ coincides with the $\mathscr{X}$-homotopy relation.

Proof. (i) $\Rightarrow$ (ii) Since $f$ is a weak equivalence iff $f$ is an isomorphism in $\mathscr{C} / \mathscr{X}$, property (i) follows directly. Property (ii) is easy to check and is left to the reader. Let $f: A \rightarrow B$ be a morphism in $\mathscr{C}$. Consider the morphisms $f_{1}:=\left(\chi^{A}, f\right): A \rightarrow X^{A} \oplus B, f_{2}:={ }^{t}\left(0,1_{B}\right): X^{A} \oplus B \rightarrow B, f_{3}:=\left(1_{A}, 0\right):$ $A \rightarrow A \oplus X_{B}, f_{4}:={ }^{t}\left(f, \chi_{B}\right): A \oplus X_{B} \rightarrow B$, where $\chi^{A}: A \rightarrow X^{A}$ is a left $\mathscr{X}$-approximation of $A$ and $\chi_{B}: X_{B} \rightarrow B$ is a right $\mathscr{X}$-approximation of $B$. Then $f=f_{1} \circ f_{2}=f_{3} \circ f_{4}$. Moreover by construction $f_{1}$ is a cofibration, $f_{4}$ is a fibration, $f_{2}$ is a trivial fibration and $f_{3}$ is a trivial cofibration. Hence property (iv) holds. It remains to prove that property (iii) is true. Consider the commutative diagram $(\dagger)$ as in definition 4.1 and assume first that $i$ is a trivial cofibration and $p$ is a fibration. By Lemma 4.4, the morphism $i$ induces a direct sum decomposition $B \cong A \oplus X$, with $X \in \mathscr{X}$. Hence without loss of generality we can assume that $B=A \oplus X$ and $i=\left(1_{A}, 0\right): A \rightarrow A \oplus X$. Then $g$ is of the form $g={ }^{t}\left(g_{1}, g_{2}\right): A \oplus X \rightarrow D$. By the commutativity of $(\dagger)$ we have $g_{1}=f \circ p$. Since $p$ is a fibration $\left(=\mathscr{X}\right.$-epic), the morphism $g_{2}$ factors through $p$. Hence there exists $\alpha: X \rightarrow C$ such that $\alpha \circ p=g_{2}$. Consider the morphism $h:={ }^{t}(f, \alpha): A \oplus X \rightarrow C$. Then $i \circ h=\left(1_{A}, 0\right) \circ{ }^{t}(f, \alpha)=f$ and $h \circ p={ }^{t}(f, \alpha) \circ p={ }^{t}(f \circ p, \alpha \circ p)={ }^{t}\left(g_{1}, g_{2}\right)=g$. A similar argument shows that the morphism $h$ exists, if $i$ is a cofibration and $p$ is a trivial fibration. Hence the triple $\left(\operatorname{Cof}_{\mathscr{X}}(\mathscr{C}), F i b_{\mathscr{X}}(\mathscr{C}), W e q_{\mathscr{X}}(\mathscr{C})\right)$ defines on $\mathscr{C}$ a closed model structure.

(ii) $\Rightarrow$ (i) Let $A$ be in $\mathscr{C}$. If $\mathscr{C}(A, X)=0, \forall X \in \mathscr{X}$, then $A \rightarrow 0$ is a left $\mathscr{X}$-approximation of $A$. Assume that there exists $X^{\prime} \in \mathscr{X}$ and a nonzero morphism $f: A \rightarrow X^{\prime}$. By Definition 4.1, there exists a factorization 
$f=f_{1} \circ f_{2}: A \stackrel{f_{1}}{\rightarrow} B \stackrel{f_{2}}{\rightarrow} X^{\prime}$ such that $f_{1}$ is a cofibration and $f_{2}$ is a trivial fibration. By the above Lemma, $f_{2}$ is a split epic with kernel in $\mathscr{X}$, hence $B \in \mathscr{X}$. Since by definition $f_{1}$ is a cofibration $=\mathscr{X}$-monic, it follows that $f_{1}$ is a left $\mathscr{X}$-appoximation of $A$. Hence $\mathscr{X}$ is covariantly finite. A similar argument shows that $\mathscr{X}$ is contravariantly finite.

By construction the projection functor $\pi: \mathscr{C} \rightarrow \mathscr{C} / \mathscr{X}$ sends weak equivalences to isomorphisms. If $F: \mathscr{C} \rightarrow \mathscr{D}$ is an additive functor such that $F(f)$ is an isomorphism, $\forall f \in W e q_{\mathscr{X}}(\mathscr{C})$, then since the morphism $X \rightarrow 0$ is a weak equivalence for any $X \in \mathscr{X}$, we have that $F(\mathscr{X})=0$. Hence there exists a unique (up to equivalence) functor $\tilde{F}: \mathscr{C} / \mathscr{X} \rightarrow \mathscr{D}$ such that $\tilde{F} \pi=F$. This shows that $\mathscr{C} / \mathscr{X}$ is equivalent to the homotopy category $\mathscr{C}\left[W e q_{\mathscr{X}}(\mathscr{C})^{-1}\right]$. Trivially all objects of $\mathscr{C}$ are fibrant and cofibrant. The assertion about homotopies is easy and is left to the reader.

If $\mathscr{X}$ is a functorially finite subcategory of an additive category $\mathscr{C}$, then we consider always $\mathscr{C}$ with the closed model structure described in the above Theorem.

If there exists a closed model structure on $\mathscr{C}$ with all objects bifibrant, then for an object $A \in \mathscr{C}, A \rightarrow 0$ is a weak equivalence iff $0 \rightarrow A$ is a weak equivalence. We call such objects acyclic and the induced full subcategory is denoted by $A c(\mathscr{C})$. One direction of the following result, first proved by Pirashvili [34] in case $\mathscr{C}$ is abelian, is a consequence of Theorem 4.5. The proof of the other direction, is similar to the proof of Theorem 4.5, using standard arguments from the theory of model categories. Since we shall not use it, its proof is left to the reader.

THEOREM 4.6. There is a bijective correspondence between closed model structures on $\mathscr{C}$ with all objects bifibrant and functorially finite subcategories of $\mathscr{C}$. The correspondence is given as follows:

$$
\begin{gathered}
(\operatorname{Cof}(\mathscr{C}), \operatorname{Fib}(\mathscr{C}), \operatorname{Weq}(\mathscr{C})) \longmapsto A c(\mathscr{C}), \\
\mathscr{X} \longmapsto\left(\operatorname{Cof}_{\mathscr{X}}(\mathscr{C}), \operatorname{Fib}_{\mathscr{X}}(\mathscr{C}), \operatorname{Weq}_{\mathscr{X}}(\mathscr{C})\right)
\end{gathered}
$$

\subsection{Homotopy pairs and Triangulations}

The following concept is fundamental in the study of stable categories.

DEFINITION 4.7. [9] A pair $(\mathscr{C}, \mathscr{X})$ of additive categories is called right (left) homotopy pair if the following conditions are true:

(1) $\mathscr{X}$ is a covariantly (contravariantly) finite full subcategory of $\mathscr{C}$.

(2) Any $\mathscr{X}$-monic ( $\mathscr{X}$-epic) has a cokernel (kernel) in $\mathscr{C}$. 
The pair $(\mathscr{C}, \mathscr{X})$ is called a homotopy pair if it is a left and right homotopy pair.

EXAMPLE 4.8. (1) Idempotent morphisms split in $\mathscr{C}$ iff $(\mathscr{C}, \mathscr{C})$ is a homotopy pair. The pair $(\mathscr{C}, 0)$ is a left (right) homotopy pair iff $\mathscr{C}$ has (co)kernels.

(2) For any abelian category $\mathscr{C}$ and any contravariantly (covariantly) finite subcategory $\mathscr{X} \subseteq \mathscr{C}$, the pair $(\mathscr{C}, \mathscr{X})$ is a left (right) homotopy pair. Here we can choose $\mathscr{X}$ to be the full subcategory of projectives (injectives), if $\mathscr{C}$ has enough of them.

(3) If $\mathscr{C}$ is a locally finitely presented additive category, then for any full subcategory $\mathscr{X} \subseteq$ f.p. $(\mathscr{C})$, the pair $(\mathscr{C}, \operatorname{Add}(\mathscr{X}))$ is a left homotopy pair. If $\mathscr{C}$ has products, then $(\mathscr{C}, \operatorname{PInj}(\mathscr{C}))$ is a right homotopy pair, where $\operatorname{PInj}(\mathscr{C})$ is the full subcategory of pure-injective objects. In particular if $\mathscr{D}$ is a skeletally small additive category with split idempotents, then $(\operatorname{Flat}(\operatorname{Mod}(\mathscr{D})), \operatorname{Proj}(\operatorname{Mod}(\mathscr{D})))$ is a left homotopy pair and if moreover $\mathscr{D}$ has weak cokernels, then $(\operatorname{Flat}(\operatorname{Mod}(\mathscr{D}))$, FlPInj $(\operatorname{Mod}(\mathscr{D})))$ is a right homotopy pair, where $\operatorname{FlPInj}(\operatorname{Mod}(\mathscr{D}))$ is the full subcategory of flat and pure-injective objects.

Usually a category carries a left and right triangulated structure in a compatible way. We formalize this situation in the following definition, see also [22].

Definition 4.9. Let $\mathscr{C}$ be an additive category. A pre-triangulation of $\mathscr{C}$ consists of the following data:

(i) An adjoint pair $(\Sigma, \Omega)$ of additive endofunctors $\Sigma, \Omega: \mathscr{C} \rightarrow \mathscr{C}$. Let $\varepsilon: \Sigma \Omega \rightarrow \operatorname{Id}_{\mathscr{C}}$ be the counit and let $\delta: \operatorname{Id}_{\mathscr{C}} \rightarrow \Omega \Sigma$ be the unit of the adjoint pair.

(ii) A collection of diagrams $\Delta$ in $\mathscr{C}$ of the form $\Omega(C) \rightarrow A \rightarrow B \rightarrow C$, such that the triple $(\mathscr{C}, \Omega, \Delta)$ is a left triangulated category.

(iii) A collection of diagrams $\nabla$ in $\mathscr{C}$ of the form $A \rightarrow B \rightarrow C \rightarrow \Sigma(A)$, such that the triple $(\mathscr{C}, \Sigma, \nabla)$ is a right triangulated category.

(iv) For any diagram in $\mathscr{C}$ with commutative left square:

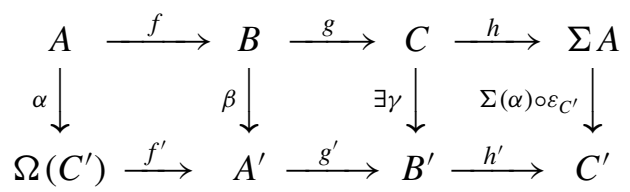

where the upper row is in $\nabla$ and the lower row is in $\Delta$, there exists a morphism $\gamma: C \rightarrow B^{\prime}$ making the diagram commutative. 
(v) For any diagram in $\mathscr{C}$ with commutative right square:

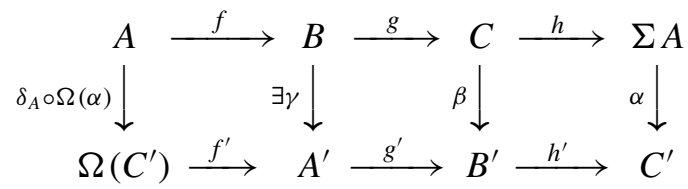

where the upper row is in $\nabla$ and the lower row is in $\Delta$, there exists a morphism $\gamma: B \rightarrow A^{\prime}$ making the diagram commutative.

A pre-triangulated category is defined to be an additive category together with a pre-triangulation.

Any right homotopy pair $(\mathscr{C}, \mathscr{X})$ induces on the stable category $\mathscr{C} / \mathscr{X}$ a right triangulated structure $\left(\Sigma_{\mathscr{X}}, \nabla_{\mathscr{X}}\right)$, where $\Sigma_{\mathscr{X}}: \mathscr{C} / \mathscr{X} \rightarrow \mathscr{C} / \mathscr{X}$ is the suspension functor and $\nabla_{\mathscr{X}}$ is the triangulation. Dually any left homotopy pair $(\mathscr{C}, \mathscr{X})$ induces on the stable category $\mathscr{C} / \mathscr{X}$ a left triangulated structure $\left(\Omega_{\mathscr{X}}, \Delta_{\mathscr{X}}\right)$, where $\Omega_{\mathscr{X}}: \mathscr{C} / \mathscr{X} \rightarrow \mathscr{C} / \mathscr{X}$ is the loop functor and $\Delta_{\mathscr{X}}$ is the triangulation, we refer to [9] for details. If $(\mathscr{C}, \mathscr{X})$ is a left (right) homotopy pair, then we consider always the stable category $\mathscr{C} / \mathscr{X}$ as a left (right) triangulated category as above and we note that in case $(\mathscr{C}, \mathscr{X})$ is a homotopy pair, then we have an adjoint pair $\left(\Sigma_{\mathscr{X}}, \Omega_{\mathscr{X}}\right)$ in $\mathscr{C} / \mathscr{X}$ [8]. In this case it is not difficult to see that the compatability conditions (iv), (v) are true. We collect the above information in the following.

COROLlary 4.10. There exists a bijective correspondence between homotopy pairs $(\mathscr{C}, \mathscr{X})$ and weak closed model structures on $\mathscr{C}$ with all objects bifibrant. The induced homotopy category $\mathscr{C} / \mathscr{X}$ is a pre-triangulated category.

In general there is no relation between the left triangulation $\Delta_{\mathscr{X}}$ and the right triangulation $\nabla_{\mathscr{X}}$ on $\mathscr{C} / \mathscr{X}$. There is a particular case in which $\Delta_{\mathscr{X}}, \nabla_{\mathscr{X}}$ are essentially the same. We recall that an additive category $\mathscr{C}$ is called Frobenius, if $\mathscr{C}$ is an exact category in the sense of Quillen, with enough projectives, enough injectives and the projectives coincide with the injectives. For instance if $\Lambda$ is an associative ring, then the category $\operatorname{Mod}(\Lambda)$ of right $\Lambda$-modules is Frobenius iff $\Lambda$ is a Quasi-Frobenius ring. If $\mathscr{P}$ is the full subcategory of projectives and $\mathscr{C}$ is Frobenius, then clearly $(\mathscr{C}, \mathscr{P})$ is a homotopy pair. We recall that a weak closed model category $\mathscr{C}$ is called stable, if the associated homotopy category $\operatorname{Ho}(\mathscr{C})$ of $\mathscr{C}$ is triangulated with the triangulation induced from the cofibrations, see [22].

COROLlary 4.11. If $\mathscr{C}$ is a Frobenius category with split idempotents, then $\mathscr{C}$ is a stable weak closed model category with cofibrations the proper monomorphisms, fibrations the proper epimorphisms and weak equivalences the stable equivalences. 
We note that many (left or right) triangulated categories arise from stable weak closed model Frobenius categories, as homotopy (a stable) categories in the above sense.This covers the stable module category of a QF-ring and the derived categroy of a ring (see [25]).

REMARK 4.12. If $(\mathscr{C}, \mathscr{X})$ is a right homotopy pair, then defining cofibrations and weak equivalences as before, it follows easily that $\mathscr{C}$ has the structure of a saturated Waldhausen category [38]. Hence by [38] the Waldhausen $K$-groups $\mathrm{K}_{n}(\mathscr{C})$ of $\mathscr{C}$ are defined, $\forall n \geq 0$. It is easy to see that $\mathrm{K}_{0}(\mathscr{C})$ is isomorphic to the Grothendieck group of the right triangulated category $\mathscr{C} / \mathscr{X}$ in the sense of [10]. This suggests that the groups $\mathrm{K}_{n}(\mathscr{C})$ can be regarded as the higher $K$-groups of the stable right triangulated category $\mathscr{C} / \mathscr{X}$.

\subsection{Brown's Abstract Homotopy Categories}

E. H. Brown in [12] formalizing the homotopy theory of CW complexes defined abstract homotopy categories. In the additive setting his definition can be formulated as follows:

Definition 4.13. An Abstract Homotopy Category is a pair $(\mathscr{C}, \mathscr{X})$ consisting of an additive category $\mathscr{C}$ and a full additive subcategory $\mathscr{X} \subseteq \mathscr{C}$, such that:

(i) $\mathscr{C}$ has coproducts and weak cokernels.

(ii) $\mathscr{X}$ is a minimal Whitehead subcategory of $\mathscr{C}$ closed under weak cokernels.

The above definition is different from that of [12] in that Brown does not require that $\mathscr{X}$ is Whitehead. In the next section we shall see many algebraic examples of abstract homotopy categories and our definition is more suitable for our purposes.

\section{Stable Module Categories}

Throughout this section $\Lambda$ will denote an associative ring. We denote by $\mathscr{P}_{\Lambda}$, resp. $\mathbf{P}_{\Lambda}$, the categories of finitely generated projective, resp. all projective, right modules, and by $\operatorname{Mod}(\Lambda)$, resp. $\bmod (\Lambda)$, the category of all, resp. finitely presented, right $\Lambda$-modules. Let $\bmod (\Lambda):=\bmod (\Lambda) / \mathscr{P}_{\Lambda}$ and $\underline{\operatorname{Mod}}(\Lambda):=$ $\operatorname{Mod}(\Lambda) / \mathbf{P}_{\Lambda}$ be the induced stable categories. Then $\left(\operatorname{Mod}(\Lambda), \mathbf{P}_{\Lambda}\right)$ is a left homotopy pair, so $\underline{\operatorname{Mod}}(\Lambda)$ is a left triangulated category with coproducts and split idempotents. Dually we denote by $\mathscr{I}_{\Lambda}$, resp. $\mathbf{I}_{\Lambda}$, the categories of finitely generated injective, resp. all injective, right modules. Let $\overline{\bmod }(\Lambda):=$ $\bmod (\Lambda) / \mathscr{I}_{\Lambda}$ and $\overline{\operatorname{Mod}}(\Lambda):=\operatorname{Mod}(\Lambda) / \mathbf{I}_{\Lambda}$ be the induced stable categories. Then $\left(\operatorname{Mod}(\Lambda), \mathbf{I}_{\Lambda}\right)$ is a right homotopy pair, so $\overline{\operatorname{Mod}}(\Lambda)$ is a right triangulated 
category with products and split idempotents. Trivially $\underline{\operatorname{Mod}}(\Lambda)$ is triangulated $\Leftrightarrow \overline{\operatorname{Mod}}(\Lambda)$ is triangulated $\Leftrightarrow \Lambda$ is a QF-ring.

Our aim in this section is to characterize when a ring $\Lambda$ has the property that the stable category $\underline{\bmod }(\Lambda), \operatorname{resp} . \overline{\bmod }(\Lambda)$, is a compact Whitehead left, resp. right, triangulated subcategory of $\operatorname{Mod}(\Lambda), \operatorname{resp} . \overline{\operatorname{Mod}}(\Lambda)$. We begin with two easy results. First we recall that a module $A$ is called FP-injective if $\mathscr{E} x t_{\Lambda}^{1}(X, A)=0$, for any finitely presented module $X$. A short exact sequence $0 \rightarrow A \rightarrow B \rightarrow C \rightarrow 0$ in $\operatorname{Mod}(\Lambda)$ is called pure if $0 \rightarrow(X, A) \rightarrow$ $(X, B) \rightarrow(X, C) \rightarrow 0$ is exact for any finitely presented right module $X$; equivalently if $0 \rightarrow A \otimes_{\Lambda} L \rightarrow B \otimes_{\Lambda} L \rightarrow C \otimes_{\Lambda} L \rightarrow 0$ is exact for any (finitely presented) left module $L$. It is well known that a module $C$, resp. $A$, is flat, resp. FP-injective, if any short exact sequence $0 \rightarrow A \rightarrow B \rightarrow C \rightarrow 0$ is pure.

Lemma 5.1. (1) $A \in \operatorname{Mod}(\Lambda)$ is flat $\Leftrightarrow \forall X \in \bmod (\Lambda):(\underline{X}, \underline{A})=0$.

(2) $A \in \operatorname{Mod}(\Lambda)$ is FP-injective $\Leftrightarrow \forall X \in \bmod (\Lambda):(\bar{X}, \bar{\Sigma}(\overline{\bar{A}}))=0$.

Proof. (1) Let $A$ be flat and let (*) : $0 \rightarrow K \rightarrow P \rightarrow A \rightarrow 0$ be exact with $P$ projective. If $X$ is finitely presented, then since $(*)$ is pure, any morphism $f: X \rightarrow A$ factors through $P$. Hence $(\underline{X}, \underline{A})=0, \forall X \in \bmod (\Lambda)$. Conversely if the last condition is true, then the sequence $(*)$ is pure. Trivially then any short exact sequence ending at $A$ is pure, so $A$ is flat. The proof of (2) is similar.

LEMMA 5.2. Let $\mathscr{C}$ be an abelian category and $\mathscr{X}$ be a full subcategory of $\mathscr{C}$.

(i) If $\mathscr{X}$ is contravariantly finite and any $\mathscr{X}$-epic is an epimorphism, then: $\mathscr{X}$ is covariantly finite (reflective) in $\mathscr{C} \Leftrightarrow \mathscr{X}$ has weak cokernels (cokernels).

(ii) If $\mathscr{X}$ is covariantly finite and any $\mathscr{X}$-monic is a monomorphism, then: $\mathscr{X}$ is contravariantly finite (coreflective) $\Leftrightarrow \mathscr{X}$ has weak kernels (kernels).

Proof. (i) Assume that $\mathscr{X}$ has weak cokernels and let $C \in \mathscr{C}$. Let $X_{1} \stackrel{f}{\rightarrow}$ $X_{0} \stackrel{\chi}{\rightarrow} C \rightarrow 0$ be an exact sequence in $\mathscr{C}$, where $X_{0} \rightarrow C$ is a right $\mathscr{X}$ approximation of $C$ and $X_{1}$ is a right $\mathscr{X}$-approximation of $\operatorname{Ker}(\chi)$. Let $g$ : $X_{0} \rightarrow X_{2}$ be a weak cokernel of $f$ in $\mathscr{X}$. Then there exists a unique morphism $\xi: C \rightarrow X_{2}$ such that $\chi \circ \xi=g$. If $h: C \rightarrow X^{\prime}$ is a morphism with $X^{\prime} \in \mathscr{X}$, then $f \circ \chi \circ h=0$, hence there exists a morphism $t: X_{2} \rightarrow X^{\prime}$ such that $\chi \circ h=g \circ t=\chi \circ \xi \circ t$. Since $\chi$ is epic, $\xi \circ t=h$. Hence $\xi$ is a left $\mathscr{X}$ approximation of $C$. Conversely if $\mathscr{X}$ is covariantly finite then by Proposition $3.1, \mathscr{X}$ has weak cokernels. The proof of the parenthetical case and of part (ii) is similar and is left to the reader. 
THEOREM 5.3. For any ring $\Lambda$ the following are equivalent.

(i) $\Lambda$ is right Noetherian.

(ii) $\left(\operatorname{Mod}(\Lambda), \mathbf{I}_{\Lambda}\right)$ is a homotopy pair.

(iii) $\mathbf{I}_{\Lambda}$ is contravariantly finite.

(iv) $\mathbf{I}_{\Lambda}$ has weak kernels.

(v) $\mathbf{I}_{\Lambda}$ is closed under coproducts.

(vi) The functor $\pi: \operatorname{Mod}(\Lambda) \rightarrow \overline{\operatorname{Mod}}(\Lambda)$ preserves coproducts.

(vii) $(\bar{X}, \bar{A})=0, \forall X \in \bmod (\Lambda)$ implies that $\bar{A}=0$, i.e. A is injective.

If $\Lambda$ is right Noetherian, then $\overline{\operatorname{Mod}}(\Lambda)$ is a pre-triangulated category with products, coproducts, weak kernels, weak cokernels (hence weak limits and weak colimits) and the suspension functor $\Sigma: \overline{\operatorname{Mod}}(\Lambda) \rightarrow \overline{\operatorname{Mod}}(\Lambda)$ has a right adjoint $\Omega_{\mathbf{I}}$.

Proof. It is well known that $\Lambda$ is right Noetherian iff any coproduct of injectives is injective, and by [8] we have that $\Lambda$ is right Noetherian iff $\mathbf{I}_{\Lambda}$ is contravariantly finite. Hence (i) is equivalent to (ii), (iii) and (v) and by Proposition 2.1, (i) is equivalent to (vi). By Lemma 5.2, (iii) is equivalent to (iv). It remains to prove that (i) is equivalent to (vii). Suppose that $\Lambda$ is right Noetherian and suppose that $(\bar{X}, \bar{A})=0$, for any finitely presented $X$. Let $I$ be a right ideal of $\Lambda$ and let $f: I \rightarrow A$ be a morphism. Since $\Lambda$ is right Noetherian, $f$ factors through the injective envelope $\mu: I \rightarrow E(I)$ of $I$ as $f=\mu \circ g$. Since $E(I)$ is injective, $\mu$ factors through the inclusion $i: I \hookrightarrow \Lambda$ as $\mu=i \circ h$. Then $f$ factors through the inclusion $i$ as $f=i \circ h \circ g$. By Baer's criterion, $A$ is injective. Conversely assume that $(\bar{X}, \bar{A})=0, \forall X \in \bmod (\Lambda)$ implies that $\bar{A}=0$. Let $\left\{E_{i} ; i \in I\right\}$ be a set of injective modules, $X$ an arbitrary finitely presented module and let $f: X \rightarrow \oplus E_{i}$ be a morphism. Since $X$ is finitely presented, the morphism $f$ factors through some finite subcoproduct $\oplus_{j \in J} E_{j},|J|<\infty$. Since $\oplus_{j \in J} E_{j}$ is injective, we have $\bar{f}=0$. By hypothesis, $\overline{\oplus E_{i}}=0$ or equivalently $\oplus E_{i}$ is injective. Hence a coproduct of injectives is injective and then $\Lambda$ is right Noetherian.

If $\Lambda$ is right Noetherian, then since $\left(\operatorname{Mod}(\Lambda), \mathbf{I}_{\Lambda}\right)$ is a homotopy pair, it follows that the suspension functor $\Sigma$ has a right adjoint $\Omega_{\mathrm{I}}$ and $\overline{\operatorname{Mod}}(\Lambda)$ is pre-triangulated by Corollary 4.10. Hence $\overline{\operatorname{Mod}}(\Lambda)$ has weak kernels and weak cokernels and has products and coproducts since $\mathbf{I}_{\Lambda}$ is closed under products and coproducts.

The following consequence is due to Pirashvili [34].

COROLlary 5.4. A ring $\Lambda$ is right Noetherian iff $\operatorname{Mod}(\Lambda)$ is a closed model category with cofibrations the monomorphisms, fibrations the $\mathbf{I}_{\Lambda}$-epimorphisms and weak equivalences the morphisms which are isomorphisms in $\overline{\operatorname{Mod}}(\Lambda)$. 
We recall that a ring $\Lambda$, resp. $\Gamma$, is called right (resp. left), Morita if there exists a ring $\Gamma$, resp. $\Lambda$, and a $\operatorname{Morita}$ duality $\bmod (\Lambda) \rightarrow \bmod \left(\Gamma^{o p}\right)$. Equivalently $\Lambda$ is right Artinian and $\operatorname{Mod}(\Lambda)$ has a finitely generated injective cogenerator.

COROLlary 5.5. For any ring $\Lambda$ the following are equivalent

(i) $\Lambda$ is right Morita.

(ii) $\Lambda$ is right Noetherian and $\bmod (\Lambda)$ is closed under injective envelopes.

(iii) $\Lambda$ is right Noetherian and $\left(\bmod (\Lambda), \mathscr{I}_{\Lambda}\right)$ is a right homotopy pair.

(iv) $\overline{\bmod }(\Lambda)$ is a (compact) Whitehead right triangulated subcategory of $\overline{\operatorname{Mod}}(\Lambda)$.

If $\Lambda$ is right Morita, then the right adjoint $\Omega_{\mathbf{I}}$ of $\Sigma$ preserves coproducts and $\Sigma$ preserves coproducts and compact objects.

Proof. (ii) $\Rightarrow$ (iv) That $\overline{\bmod }(\Lambda)$ is a Whitehead subacategory, follows from [25] (actually in [25] is assumed that $\Lambda$ is right Artinian, but the proof works in our case). By hypothesis, $\bmod (\Lambda)$ is abelian with enough injectives, so $\overline{\bmod }(\Lambda)$ is a right triangulated subcategory of $\overline{\operatorname{Mod}}(\Lambda)$ which obviously consists of compact objects.

(iv) $\Rightarrow$ (ii) Let $A$ be a right module and suppose that $(\bar{X}, \bar{A})=0$, for any finitely presented $X$. Then $\left(\bar{X}, 0_{\bar{A}}\right)$ is an isomorphism. Since $\overline{\bmod }(\Lambda)$ is a Whitehead subcategory, $0_{\bar{A}}$ is an isomorphism, so $\bar{A}=0$. By Theorem 5.3, $\Lambda$ is right Noetherian. Since $\overline{\bmod }(\Lambda)$ is a right triangulated subcategory of $\overline{\operatorname{Mod}}(\Lambda)$, its follows that for any finitely presented $X$, its suspension $\Sigma(X)$ is finitely presented and this implies that the injective envelope of $X$ is also finitely presented. Obviously (ii) is equivalent to (iii) and (i) implies (ii). Conversely if (ii) holds, then the injective envelope of $\Lambda$ is finitely generated. This implies that $\Lambda$ is right Artinian and the direct sum of the injective envelopes of the isoclasses of simple modules is a finitely generated injective cogenerator, so $\Lambda$ is right Morita.

If $\left\{A_{j} ; i \in J\right\}$ is a set of right $\Lambda$-modules, and $0 \rightarrow K_{j} \rightarrow I_{j} \rightarrow A_{j}$ are exact sequences where $I_{j}$ are $\mathbf{I}_{\Lambda}$-approximations of $A_{j}$, then using the hypothesis (i), it is not difficult to see that $\oplus I_{j}$ is a right $\mathbf{I}_{\Lambda}$-approximation of $\oplus A_{j}$. By the construction of $\Omega_{\mathbf{I}}$ in [8], this implies that $\Omega_{\mathbf{I}}\left(\oplus \underline{A}_{j}\right)=\oplus \underline{K}_{j}=$ $\oplus \Omega_{\mathbf{I}}\left(\underline{A}_{j}\right)$. Hence $\Omega_{\mathbf{I}}$ preserves coproducts. Then $\Sigma$ preserves compact objects by Corollary 3.12.

Now we turn our attention to the stable category modulo projectives. First we need a simple observation.

REMARK 5.6. It is easy to see that: $\Lambda$ is left coherent $\Leftrightarrow \mathscr{P}_{\Lambda}$ has weak cokernels $\Leftrightarrow \mathscr{P}_{\Lambda^{o p}}$ has weak kernels $\Leftrightarrow \mathscr{P}_{\Lambda}$ is covariantly finite in $\bmod (\Lambda)$ 
$\Leftrightarrow\left(\bmod (\Lambda), \mathscr{P}_{\Lambda}\right)$ is a right homotopy pair $\Leftrightarrow\left(\bmod \left(\Lambda^{o p}\right), \mathscr{P}_{\Lambda^{o p}}\right)$ is a left homotopy pair. In this case by subsection 4.2 , the stable category $\underline{\bmod }(\Lambda)$ is right triangulated and the stable category $\underline{\bmod }\left(\Lambda^{o p}\right)$ is left triangulated. Hence: $\Lambda$ is left and right coherent $\Leftrightarrow\left(\bmod (\Lambda), \mathscr{P}_{\Lambda}\right),\left(\bmod \left(\Lambda^{o p}\right), \mathscr{P}_{\Lambda^{o p}}\right)$ are homotopy pairs. In this case the categories $\bmod (\Lambda), \bmod \left(\Lambda^{o p}\right)$ are weak closed model categories and the associated homotopy categories $\bmod (\Lambda), \bmod \left(\Lambda^{o p}\right)$ are pre-triangulated.

We have the following characterization of left coherent and right perfect rings.

THEOREM 5.7. For any ring $\Lambda$ the following are equivalent.

(i) $\Lambda$ is left coherent and right perfect.

(ii) $\left(\operatorname{Mod}(\Lambda), \mathbf{P}_{\Lambda}\right)$ is a homotopy pair.

(iii) $\mathbf{P}_{\Lambda}$ is covariantly finite.

(iv) $\mathbf{P}_{\Lambda}$ has weak cokernels.

(v) $\mathbf{P}_{\Lambda}$ is closed under products.

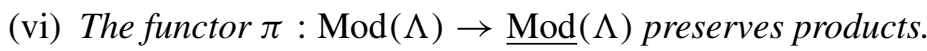

(vii) The following are true:

(a) $\left(\bmod (\Lambda), \mathscr{P}_{\Lambda}\right)$ is a right homotopy pair.

(b) $(\underline{X}, \underline{A})=0, \forall X \in \bmod (\Lambda)$ implies that $\underline{A}=0$, i.e. A is projective.

(viii) The following are true:

(a) $\left(\bmod (\Lambda), \mathscr{P}_{\Lambda}\right)$ is a right homotopy pair.

(b) $\bmod (\Lambda)$ is (compact) Whitehead right triangulated subcategory of $\operatorname{Mod}(\Lambda)$.

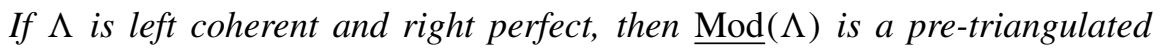
category with products, coproducts, weak kernels, weak cokernels (hence weak limits and weak colimits) and the loop functor $\Omega: \operatorname{Mod}(\Lambda) \rightarrow \underline{\operatorname{Mod}}(\Lambda)$ preserves coproducts and has a left adjoint $\Sigma_{\mathbf{P}}$ which preserves coproducts and compact objects.

Proof. The equivalence (i) $\Leftrightarrow$ (v) is a well-known result of Chase. The equivalence (iii) $\Leftrightarrow$ (v) follows from Proposition 2.5. The equivalence (ii) $\Leftrightarrow$ (iii) is trivial. The equivalence (v) $\Leftrightarrow$ (vi) follows from Proposition 2.1. The equivalence (iii) $\Leftrightarrow$ (iv) follows from Lemma 5.2. The equivalence (i) $\Leftrightarrow$ (vii) follows from the above Remark and Lemma 5.1. It remains to show that (i) is equivalent to (viii).

(i) $\Rightarrow$ (viii) Assume that $\Lambda$ is left coherent and right perfect. From part

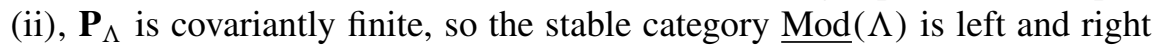


triangulated with loop functor $\Omega$ and suspension functor its adjoint $\Sigma_{\mathbf{P}}$, see [8]. Let $f: A \rightarrow B$ be a morphism such that $(\underline{X}, f):(\underline{X}, \underline{A}) \rightarrow(\underline{X}, \underline{B})$ is an isomorphism. If $p_{B}: P_{B} \rightarrow B$ is the projective cover of $B$, we have a short exact sequence $(*): 0 \rightarrow C \stackrel{(g, k)}{\longrightarrow} A \oplus P_{B} \stackrel{{ }^{t}\left(f, p_{B}\right)}{\longrightarrow} B \rightarrow 0$, which induces a triangle $\Omega(\underline{B}) \stackrel{h}{\rightarrow} \underline{C} \stackrel{g}{\rightarrow} \underline{A} \stackrel{f}{\rightarrow} \underline{B}$. Then $\forall X \in \bmod (\Lambda)$, we have a long exact sequence $\cdots \rightarrow(\underline{X}, \Omega(\underline{A})) \stackrel{(\underline{X}, \Omega(\underline{f}))}{\longrightarrow}(\underline{X}, \Omega(\underline{B})) \stackrel{(\underline{X}, \underline{h})}{\longrightarrow}(\underline{X}, \underline{C}) \stackrel{(\underline{X}, \underline{g})}{\longrightarrow}$ $(\underline{X}, \underline{A}) \stackrel{(\underline{X}, \underline{f})}{\longrightarrow}(\underline{X}, \underline{B})$. Using the adjoint pair $\left(\Sigma_{\mathbf{P}}, \Omega\right)$, we have the following commutative diagram:

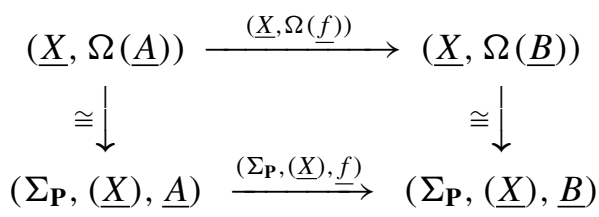

By the above remark, $\Sigma_{\mathbf{P}}(\underline{X})$ is finitely presented, hence $\left(\Sigma_{\mathbf{P}}(\underline{X}), f\right)$ and equivalently $(\underline{X}, \Omega(\underline{f}))$ is an isomorphism. We deduce that $\forall X \in \bmod (\Lambda)$ : $(\underline{X}, \underline{C})=0$. Then from part (vi), $\underline{C}=0$, so $C$ is projective. We claim that $(*)$ is pure. Indeed if $\alpha: X \rightarrow B$ is a morphism with $X$ finitely presented, then since $(\underline{X}, f)$ is an isomorphism, there exists $\beta: \underline{X} \rightarrow \underline{A}$ such that $\beta \circ f=\underline{\alpha}$. This means that $\alpha-\beta \circ f$ factors through $p_{B}$. Hence there exists $t: X \rightarrow P_{B}$ with $\alpha-\beta \circ f=t \circ p_{B}$. This trivially implies that $\alpha$ factors through ${ }^{t}\left(f, p_{B}\right)$. Hence $(*)$ is a pure exact sequence. Since $\Lambda$ is right perfect, by [24] any projective is pure injective, hence $C$ is pure injective. We conclude that $(*)$ splits. Then obviously $f$ is an isomorphism.

(viii) $\Rightarrow$ (i) Suppose that $(\underline{X}, \underline{A})=0, \forall X \in \bmod (\Lambda)$. Then $\left(\underline{X}, 0_{A}\right)$ is an isomorphism $\forall X \in \bmod (\Lambda)$. Since $\underline{\bmod }(\Lambda)$ is Whitehead, $0_{\underline{A}}$ is an isomorphism, so $\underline{A}=0$. By Lemma $5.1, \Lambda$ is right perfect. Since $\mathscr{P}_{\Lambda}$ is covariantly finite in $\bmod (\Lambda), \Lambda$ is left coherent, by the above remark.

The following consequences are due to Pirashvili [34].

COROLlaRY 5.8. A ring $\Lambda$ is left coherent and right perfect iff $\operatorname{Mod}(\Lambda)$ is a closed model category with fibrations the epimorphisms, cofibrations the $\mathbf{P}_{\Lambda}$-mono-morphisms and weak equivalences the stable equivalences, i.e. the morphisms which are isomorphisms in $\underline{\operatorname{Mod}}(\Lambda)$.

Corollary 5.9. A ring $\Lambda$ is Quasi-Frobenius iff $\operatorname{Mod}(\Lambda)$ is a stable closed model category with fibrations the epimorphisms, cofibrations the monomorphisms and weak equivalences the stable equivalences, i.e. the morphisms which are isomorphisms in $\underline{\operatorname{Mod}}(\Lambda)$ or in $\overline{\operatorname{Mod}}(\Lambda)$.

Since $\Lambda$ is right Artinian iff $\Lambda$ is right Noetherian and left perfect, we have the following consequence of Theorems 5.3 and 5.7. 
COROLlary 5.10. The following are equivalent:

(i) $\Lambda$ is right Artinian.

(ii) $\left(\operatorname{Mod}(\Lambda), \mathbf{I}_{\Lambda}\right)$ and $\left(\operatorname{Mod}\left(\Lambda^{o p}\right), \mathbf{P}_{\Lambda^{o p}}\right)$ are homotopy pairs.

$\Lambda$ is Artinian $\Leftrightarrow$ the following are homotopy pairs:

$\left(\operatorname{Mod}(\Lambda), \mathbf{I}_{\Lambda}\right),\left(\operatorname{Mod}(\Lambda), \mathbf{P}_{\Lambda}\right),\left(\operatorname{Mod}\left(\Lambda^{o p}\right), \mathbf{I}_{\Lambda^{o p}}\right),\left(\operatorname{Mod}\left(\Lambda^{o p}\right), \mathbf{P}_{\Lambda^{o p}}\right)$.

If $\Lambda$ is right Noetherian, then $\overline{\operatorname{Mod}}(\Lambda)$ as a pre-triangulated category is left and right triangulated. We use the notation $\operatorname{Mod}_{R}(\Lambda)$ when we consider $\overline{\operatorname{Mod}}(\Lambda)$ as a right triangulated category with suspension functor $\Sigma$ and the notation $\overline{\operatorname{Mod}}_{L}(\Lambda)$ when we consider $\overline{\operatorname{Mod}}(\Lambda)$ as a left triangulated category with loop functor the right adjoint $\Omega_{\mathbf{I}}$ of $\Sigma$. Similarly if $\Lambda$ is left coherent and right perfect, then $\underline{\operatorname{Mod}}(\Lambda)$ as a pre-triangulated category is left and right triangulated. We use the notation $\underline{\operatorname{Mod}}^{\mathrm{L}}(\Lambda)$ when we consider $\underline{\operatorname{Mod}}(\Lambda)$ as a left triangulated category with loop functor $\Omega$ and the notation $\underline{\operatorname{Mod}}^{\mathrm{R}}(\Lambda)$ when we consider $\underline{\operatorname{Mod}}(\Lambda)$ as a right triangulated category with suspension functor the left adjoint $\Sigma_{\mathbf{P}}$ of $\Omega$.

The next three Corollaries are direct consequences of our previous results.

COROLlary 5.11. The following statements are equivalent.

(i) $\Lambda$ is a right Morita ring.

(ii) The pair $(\overline{\operatorname{Mod}}(\Lambda), \overline{\bmod }(\Lambda))$ is an Abstract Homotopy Category.

(iii) The pair $\left(\mathbf{I}_{\Lambda}, \mathscr{I}_{\Lambda}\right)$ is an Abstract Homotopy Category.

If $\Lambda$ is right Morita, then we have the following.

$(\alpha)$ The right triangulated category $\overline{\operatorname{Mod}}_{\mathrm{R}}(\Lambda)$ is compactly generated with minimal compact Whitehead generating subcategory $\overline{\bmod }(\Lambda)$.

( $\beta$ ) An additive functor $F: \overline{\operatorname{Mod}}_{R}(\Lambda)^{o p} \rightarrow \mathscr{A} b$ is representable iff $F$ is cohomological and sends coproducts to products.

COROLlary 5.12. The following statements are equivalent.

(i) $\Lambda$ is a left coherent and right perfect ring.

(ii) The pair $(\underline{\operatorname{Mod}}(\Lambda), \underline{\bmod }(\Lambda))$ is an Abstract Homotopy Category.

(iii) The pair $\left(\mathbf{P}_{\Lambda}, \mathscr{P}_{\Lambda}\right)$ is an Abstract Homotopy Category.

If $\Lambda$ is left coherent and right perfect, then we have the following.

$(\alpha)$ The right triangulated category $\operatorname{Mod}^{\mathrm{R}}(\Lambda)$ is compactly generated with minimal compact Whitehead generating subcategory $\bmod (\Lambda)$.

( $\beta$ ) An additive functor $F: \underline{\operatorname{Mod}}^{\mathrm{R}}(\Lambda)^{o p} \rightarrow \mathscr{A} b$ is representable iff $F$ is cohomological and sends coproducts to products. 
COROLlaRy 5.13. If there exists a Morita duality $\mathrm{D}: \bmod (\Lambda) \rightarrow \bmod \left(\Gamma^{o p}\right)$, then:

(i) $(\overline{\operatorname{Mod}}(\Lambda), \overline{\bmod }(\Lambda))$ and $\left(\underline{\operatorname{Mod}}\left(\Lambda^{o p}\right), \underline{\bmod }\left(\Lambda^{o p}\right)\right)$ are Abstract Homotopy Categories.

(ii) $\left(\overline{\operatorname{Mod}}\left(\Gamma^{o p}\right), \overline{\bmod }\left(\Gamma^{o p}\right)\right)$ and $(\underline{\operatorname{Mod}}(\Gamma), \underline{\bmod }(\Gamma))$ are Abstract Homotopy Categories.

Remark 5.14. Let PProj $(\Lambda), \operatorname{PInj}(\Lambda)$ be the full subcategories of $\operatorname{Mod}(\Lambda)$ consisting of all pure-projective, resp. pure-injective modules. The following well known result shows that the pure version of the above theory is trivial.

The following statements are equivalent:

(i) The ring $\Lambda$ is right pure semisimple.

(ii) $\operatorname{PProj}(\Lambda)$ is covariantly finite.

(iii) $\operatorname{PInj}(\Lambda)$ is contravariantly finite.

(iv) $\operatorname{PProj}(\Lambda)$ has weak cokernels.

(v) $\operatorname{PInj}(\Lambda)$ has weak kernels.

(vi) $\operatorname{PProj}(\Lambda)$ is closed under products.

(vii) $\operatorname{PInj}(\Lambda)$ is closed under coproducts.

(viii) The stable category $\operatorname{Mod}(\Lambda) / \operatorname{PProj}(\Lambda)=0$.

(ix) The stable category $\operatorname{Mod}(\Lambda) / \operatorname{PInj}(\Lambda)=0$.

(x) Any pure-projective is pure-injective.

(xi) Any pure-injective is pure-projective.

(xii) The functor category $\operatorname{Mod}(\bmod (\Lambda))$ is perfect.

(xiii) The functor category $\operatorname{Mod}\left(\bmod (\Lambda)^{o p}\right)$ is locally Noetherian.

We close this section with the following applications of our previous results.

Corollary 5.15. (1) $\Lambda$ is right hereditary and right Noetherian $\Leftrightarrow$ the canonical functor $\pi: \operatorname{Mod}(\Lambda) \rightarrow \overline{\operatorname{Mod}}(\Lambda)$ has a right adjoint $\Leftrightarrow \mathbf{I}_{\Lambda}$ is a torsion class.

(2) $\Lambda$ is left coherent right perfect and right hereditary $\Leftrightarrow$ the canonical functor $\pi: \operatorname{Mod}(\Lambda) \rightarrow \underline{\operatorname{Mod}}(\Lambda)$ has a left adjoint $\Leftrightarrow \mathbf{P}_{\Lambda}$ is a torsion-free class.

Proof. (1) If $\pi$ has a right adjoint, then $\pi$ preserves cokernels and coproducts. Hence by Proposition 2.1, $\mathbf{I}_{\Lambda}$ is closed under coproducts and $\Lambda$ is right Noetherian. Let $0 \rightarrow A \rightarrow I(A) \rightarrow \Sigma(A) \rightarrow 0$ be an exact sequence, where $I(A)$ is the injective envelope of $A$. Since $\pi$ preserves cokernels, it follows directly that $\Sigma(\bar{A})=0$ and this implies that $\Lambda$ is right hereditary. Conversely 
if $\Lambda$ is right hereditary and right Noetherian then it is easy to see that $\pi$ preserves coproducts and weak cokernels. Then by Theorem 3.8, $\pi$ has a right adjoint. Trivially if $\Lambda$ is right Noetherian and right hereditary, then $\mathbf{I}_{\Lambda}$ is a torsion class with torsion-free class the full subcategory $\mathscr{R}$ of modules without injective summands. If $\mathbf{I}_{\Lambda}$ is a torsion class, then obviously $\pi: \mathscr{R} \rightarrow \underline{\operatorname{Mod}}(\Lambda)$ is an equivalence. Hence $\pi$ has a right adjoint, since $\mathscr{R}$ is reflective. Part (2) is similar and is left to the reader.

Corollary 5.16. (1) $\Lambda$ is right Noetherian and r.gl.dim $\Lambda \leq 2$ iff $\mathbf{I}_{\Lambda}$ is a coreflective subcategory of $\operatorname{Mod}(\Lambda)$.

(2) $\Lambda$ is left coherent right perfect and $\mathrm{r} . \mathrm{gl} \cdot \operatorname{dim} \Lambda \leq 2$ iff $\mathbf{P}_{\Lambda}$ is a reflective subcategory of $\operatorname{Mod}(\Lambda)$.

Proof. See [5] or use Corollaries 5.11, 5.12 and Theorem 3.8.

\section{Stable Homotopy Categories}

\subsection{Stable Homotopy Categories}

We recall [8], [19], [27], that given a left or right triangulated category $\mathscr{C}$, there exists a triangulated category $\mathscr{S}(\mathscr{C})$ and an exact functor $\mathbf{S}: \mathscr{C} \rightarrow \mathscr{S}(\mathscr{C})$, which is universal for exact functors from $\mathscr{C}$ to triangulated categories. In other words the pair $(\mathbf{S}, \mathscr{S}(\mathscr{C}))$ satisfies the following universal property: if $F: \mathscr{C} \rightarrow \mathscr{D}$ is an exact functor to a triangulated category $\mathscr{D}$, then there exists a unique exact functor $F^{!}: \mathscr{S}(\mathscr{C}) \rightarrow \mathscr{D}$, such that $F^{!} \mathbf{S}=F$. The triangulated category $\mathscr{S}(\mathscr{C})$ is the stabilization of $\mathscr{C}$ and the functor $\mathbf{S}$ is the stabilization functor. Obviously $\mathscr{C}$ is triangulated iff the stabilization functor $\mathbf{S}$ is a triangle equivalence. The stabilization of $\mathscr{C}$ is trivial iff the loop or suspension functor is locally nilpotent. We refer to [8] for the description and further information on the stabilization construction.

Definition 6.1. Let $\mathscr{C}$ be a left or right triangulated category. We say that $\mathscr{C}$ admits a stable homotopy category if its stabilization $\mathscr{S}(\mathscr{C})$ is compactly generated.

Definition 6.2. (i) A ring $\Lambda$ has a (right) projective stable homotopy category if the left triangulated category $\underline{\operatorname{Mod}}(\Lambda)$ admits a stable homotopy category.

(ii) A ring $\Lambda$ has a (right) injective stable homotopy category if the right triangulated category $\overline{\operatorname{Mod}}(\Lambda)$ admits a stable homotopy category.

ExAmPLe 6.3. If $\Lambda$ is a QF-ring, then $\Lambda$ has a left and right projective and injective stable homotopy category. Indeed by Corollaries 5.9, 5.12, $\underline{\operatorname{Mod}}(\Lambda)=\overline{\operatorname{Mod}}(\Lambda)$ is a compactly generated triangulated category, and coincides with its stabilization. 
REMARK 6.4. The projective stable homotopy category is invariant under derived equivalence. Indeed if $\Lambda, \Gamma$ are derived equivalent rings, then the stabilization of $\underline{\operatorname{Mod}}(\Lambda)$ is triangle equivalent to the stabilization of $\underline{\operatorname{Mod}}(\Gamma)$. Hence $\Lambda$ has a projective stable homotopy category iff $\Gamma$ has a projective stable homotopy category.

Our aim in this section is to study rings which admit a projective or injective stable homotopy category. Before we proceed further we need some definitions and results from [8]. First we recall that a right $\Lambda$-module $A$ is called Gorenstein-projective if there exists an exact sequence $\cdots \rightarrow P^{-1} \rightarrow$ $P^{0} \rightarrow P^{1} \rightarrow \cdots$ of projective modules with $\operatorname{Im}\left(P^{-1} \rightarrow P^{0}\right)=A$ and the sequence remains exact applying $\operatorname{Hom}_{\Lambda}\left(-, \mathbf{P}_{\Lambda}\right)$. The full subcategory of all Gorenstein-projective modules is denoted by $\mathscr{G}_{\mathbf{P}}(\operatorname{Mod}(\Lambda))$, and the induced stable category modulo projectives is denoted by $\mathscr{G}_{\mathbf{P}}(\operatorname{Mod}(\Lambda))$. The full subcategory $\mathscr{G}_{\mathbb{I}}(\operatorname{Mod}(\Lambda))$ of Gorenstein-injective modules is defined dually and the induced stable category modulo injectives is denoted by $\mathscr{G}_{\mathbb{I}}(\overline{\operatorname{Mod}}(\Lambda))$. By the results of [8], the stable category $\mathscr{G}_{\mathbf{P}}(\operatorname{Mod}(\Lambda))$ is a full triangulated subcategory of $\operatorname{Mod}^{L}(\Lambda)$ and dually the stable category $\mathscr{G}_{\mathbf{I}}(\overline{\operatorname{Mod}}(\Lambda))$ is a full triangulated subcategory of $\overline{\operatorname{Mod}}_{R}(\Lambda)$. We denote by $\mathscr{G}_{\mathbf{P}}(\underline{\bmod }(\Lambda))$, resp. $\mathscr{G}_{\mathbf{I}}(\overline{\bmod }(\Lambda))$, the full subcategory of $\mathscr{G}_{\mathbf{P}}(\operatorname{Mod}(\Lambda))$, resp. $\mathscr{G}_{\mathbf{I}}(\overline{\operatorname{Mod}}(\Lambda))$, consisting of finitely presented modules. Note that $\mathscr{G}_{\mathbf{P}}(\operatorname{Mod}(\Lambda)), \mathscr{G}_{\mathbf{I}}(\operatorname{Mod}(\Lambda))$ are Frobenius weak closed model categories. Obviously if $\Lambda$ is a QF-ring then $\mathscr{G}_{\mathbf{P}}(\operatorname{Mod}(\Lambda))=$ $\operatorname{Mod}(\Lambda)=\mathscr{G}_{\mathbf{I}}(\operatorname{Mod}(\Lambda))$. If any module has finite projective, resp. injective, dimension, then $\mathscr{G}_{\mathbf{P}}(\operatorname{Mod}(\Lambda))=\mathbf{P}_{\Lambda}$, resp. $\mathscr{G}_{\mathbf{I}}(\operatorname{Mod}(\Lambda))=\mathbf{I}_{\Lambda}$. If $\Lambda$ is Noetherian (or coherent) ring, the Gorenstein-projective modules $\mathscr{G}_{\mathscr{P}}(\bmod (\Lambda))$, are exactly the modules in $\bmod (\Lambda)$ with zero G-dimension in the sense of Auslander-Bridger [1].

Definition 6.5. [8] A ring $\Lambda$ is called right Gorenstein if any projective right module has finite injective dimension and any injective right module has finite projective dimension. If $\Lambda$ is right Gorenstein, then $\sup \{$ p.d.I; $I \in$ $\left.\mathbf{I}_{\Lambda}\right\}=\sup \left\{\right.$ i.d. $\left.P ; P \in \mathbf{P}_{\Lambda}\right\}<\infty$. This number is called the (right) Gorenstein dimension of $\Lambda$ and is denoted by $\mathrm{G}-\operatorname{dim} \Lambda$.

We note that the Gorenstein property of a ring is a necessary and sufficient condition for the existence of Tate-Vogel (co)homology and complete projective or injective resolutions [8]. We need the following basic result from [8].

Lemma 6.6. Let $\Lambda$ be a right Gorenstein ring with $\mathrm{G}-\operatorname{dim} \Lambda=d$.

(1) The stabilization of $\overline{\operatorname{Mod}}_{R}(\Lambda)$ is the stable category $\mathscr{G}_{\mathbf{I}}(\overline{\operatorname{Mod}}(\Lambda))$ with stabilization functor $\Sigma^{-d} \Sigma^{d}: \overline{\operatorname{Mod}}_{R}(\Lambda) \rightarrow \mathscr{G}_{\mathbf{I}}(\overline{\operatorname{Mod}}(\Lambda))$. 
(2) The stabilization of $\underline{\operatorname{Mod}}^{\mathrm{L}}(\Lambda)$ is the stable category $\mathscr{G}_{\mathbf{P}}(\underline{\operatorname{Mod}}(\Lambda))$ with stabilization functor $\Omega^{-d} \Omega^{d}: \underline{\operatorname{Mod}}^{\mathrm{L}}(\Lambda) \rightarrow \mathscr{G}_{\mathbf{P}}(\underline{\operatorname{Mod}}(\Lambda))$.

(3) $\Omega^{d}(\operatorname{Mod}(\Lambda))=\mathscr{G}_{\mathbf{P}}(\operatorname{Mod}(\Lambda)), \Sigma^{d}(\operatorname{Mod}(\Lambda))=\mathscr{G}_{\mathbf{I}}(\operatorname{Mod}(\Lambda))$ and there exists a triangle equivalence $\mathscr{G}_{\mathbf{P}}(\underline{\operatorname{Mod}}(\Lambda)) \approx \mathscr{G}_{\mathbf{I}}(\overline{\operatorname{Mod}}(\Lambda))$.

Our first main result in this section is the following.

THEOREM 6.7. Any left coherent and right perfect or right Morita right Gorenstein ring, has a (right) projective and injective stable homotopy category.

Proof. By the above Lemma it suffices to show that the triangulated category $\mathscr{G}_{\mathbf{P}}(\underline{\operatorname{Mod}}(\Lambda))$ or equivalently $\mathscr{G}_{\mathbf{I}}(\overline{\operatorname{Mod}}(\Lambda))$ is compactly generated. Suppose first that $\Lambda$ is coherent and right perfect. Obviously $\mathscr{G}_{\mathbf{P}}(\underline{\operatorname{Mod}}(\Lambda))$ has coproducts. Let $A$ be a Gorenstein-projective module and suppose that $\forall \underline{G} \in$ $\mathscr{G}_{\mathscr{P}}(\underline{\bmod }(\Lambda))$ we have $(\underline{G}, \underline{A})=0$. Then any morphism from a finitely presented Gorenstein-projective module to $A$ factors through a projective module. Since $\Lambda$ is left coherent and right perfect by [8] we have that $\mathscr{G}_{\mathbf{P}}(\operatorname{Mod}(\Lambda))$ is covariantly finite in $\operatorname{Mod}(\Lambda)$ and it is not difficult to see that if $X$ is finitely presented then the left $\mathscr{G}_{\mathbf{P}}(\operatorname{Mod}(\Lambda))$-approximation of $X$ is finitely presented. Now let $X$ be an arbitrary finitely presented module and $f: X \rightarrow A$ be a morphism. Let $g^{X}: X \rightarrow G^{X}$ be its left Gorenstein-projective approximation. Since $A$ is Gorenstein-projective, the morphism $f$ factors through $g^{X}$. Hence there exists $h: G^{X} \rightarrow A$ such that $g^{X} \circ h=f$. Since $G^{X}$ is in $\mathscr{G}_{\mathscr{P}}(\bmod (\Lambda))$, the morphism $h$ factors through a projective. This implies that the morphism $f$ factors through a projective module. This shows that $(\underline{X}, \underline{A})=0, \forall X \in \bmod (\Lambda)$. By Theorem 5.7, we have that $A$ is projective, i.e. $\underline{A}=0$. We conclude that $\forall \underline{G} \in \mathscr{G}_{\mathscr{P}}(\underline{\bmod }(\Lambda)):(\underline{G}, \underline{A})=0 \Rightarrow \underline{A}=0$. Since $\mathscr{G}_{\mathscr{P}}(\underline{\bmod }(\Lambda))$ is skeletally small triangulated and consists of compact objects, by Corollary 3.17 it follows that the triangulated category $\mathscr{G}_{\mathbf{P}}(\operatorname{Mod}(\Lambda))$ is compactly generated. Since by Lemma $6.6, \mathscr{G}_{\mathbf{P}}(\underline{\operatorname{Mod}}(\Lambda))$ is triangle equivalent to $\mathscr{G}_{\mathbf{I}}(\overline{\operatorname{Mod}}(\Lambda))$, the latter is also compactly generated. If $\Lambda$ is right Morita, the proof is similar and is left to the reader.

COROLlary 6.8. Let $\Lambda$ be an Artin algebra of finite selfinjective dimension, i.e. i.d. $\Lambda_{\Lambda}<\infty$ and i.d. $\Lambda_{\Lambda^{o p}}^{o p}<\infty$. Then $\Lambda$ has a left and right projective and injective stable homotopy category.

Proof. Follows from Theorem 6.7, since a Noetherian ring $\Lambda$ is left Gorenstein iff $\Lambda$ is right Gorenstein iff i.d. $\Lambda_{\Lambda}<\infty$ and i.d. $\Lambda_{\Lambda^{o p}}^{o p}<\infty$, see [8].

COROLlARY 6.9. Let $\Lambda$ be a left coherent and right perfect right Gorenstein ring and let $\left\{H^{n} ; h^{n}\right\}_{n \in Z}$ be a cohomology theory in $\underline{\operatorname{Mod}}^{\mathrm{R}}(\Lambda)$. Then each $H^{n}$ 
sends coproducts to products $\Leftrightarrow$ there exists a Gorenstein-projective module A such that $H^{n} \cong \underline{\operatorname{Mod}}(\Lambda)\left(-, \Omega^{-n}(\underline{A})\right)$. Hence with the notation of section 3 :

$$
\widehat{\operatorname{CohTh}}\left(\operatorname{Mod}^{\mathrm{R}}(\Lambda)\right) \approx \mathscr{G}_{\mathbf{P}}(\underline{\operatorname{Mod}}(\Lambda)) .
$$

Proof. By Theorem 3.21, $\widehat{\operatorname{CohTh}}\left(\operatorname{Mod}^{\mathrm{R}}(\Lambda)\right)$ is equivalent to the costabilization of $\operatorname{Mod}^{L}(\Lambda)$. Since $\Lambda$ is right Gorenstein, by [8] this costabilization is triangle equivalent to the stabilization $\mathscr{G}_{\mathbf{P}}(\operatorname{Mod}(\Lambda))$.

A similar result holds for cohomology theories defined over $\overline{\operatorname{Mod}}_{R}(\Lambda)$. If $\Lambda$ is left coherent and right perfect, resp. right Morita, then by Corollary 5.12, resp. Corollary 5.11, we know that cohomological functors sending coproducts to products defined over $\operatorname{Mod}^{R}(\Lambda)$, resp. $\overline{\operatorname{Mod}}_{R}(\Lambda)$, are representable. The following result shows what happens in the Gorenstein case, for the remaining triangulations. First we need the following description of compacts objects.

LEMMA 6.10. Under the assumptions of Theorem 6.7, we have:

$$
\mathscr{G}_{\mathbf{P}}(\underline{\operatorname{Mod}}(\Lambda))^{b}=\mathscr{G}_{\mathscr{P}}(\underline{\bmod }(\Lambda)) \text { and } \mathscr{G}_{\mathbf{I}}(\overline{\operatorname{Mod}}(\Lambda))^{b}=\mathscr{G}_{\mathscr{I}}(\overline{\bmod }(\Lambda)) \text {. }
$$

In particular if $\Lambda$ is Quasi-Frobenius, then: $\underline{\operatorname{Mod}}(\Lambda)^{b}=\underline{\bmod }(\Lambda)$.

Proof. Since any finitely presented module becomes compact in the stable categories, we have $\mathscr{G}_{\mathscr{P}}(\bmod (\Lambda)) \subseteq \mathscr{G}_{\mathbf{P}}(\operatorname{Mod}(\Lambda))^{b}$. By the above Theorem, $\mathscr{G}_{\mathscr{P}}(\underline{\bmod }(\Lambda))$ is a skeletally small generating epaisse subcategory of $\mathscr{G}_{\mathbf{P}}(\operatorname{Mod}(\Lambda))$. Then the assertion follows from Neeman-Ravenel Theorem (see Theorem 5.3 in [26]).

COROLlary 6.11. ( $\alpha)$ Let $\Lambda$ be a left coherent right perfect right Gorenstein ring.

(i) Let $H: \operatorname{Mod}^{\mathrm{L}}(\Lambda)^{o p} \rightarrow \mathscr{A} b$ be a cohomological functor which sends coproducts to products. Then there exists a Gorenstein-projective module $G$ such that $\left.H\right|_{\mathscr{G}_{\mathrm{P}}(\operatorname{Mod}(\Lambda))}=(-, \underline{G})$.

(ii) Let $F: \underline{\operatorname{Mod}}^{L}(\Lambda) \rightarrow \mathscr{A} b$ be a homological functor which preserves products and coproducts. Then there exists a finitely presented Gorensteinprojective module $G$ such that $\left.H\right|_{\mathscr{S}_{\mathrm{p}}(\operatorname{Mod}(\Lambda))}=(\underline{G},-)$.

( $\beta$ ) Let $\Lambda$ be a right Morita right Gorenstein ring.

(i) Let $H: \overline{\operatorname{Mod}}_{\mathrm{L}}(\Lambda)^{o p} \rightarrow \mathscr{A} b$ be a cohomological functor which sends coproducts to products. Then there exists a Gorenstein-injective module $G$ such that $\left.H\right|_{\mathscr{G}_{1}(\overline{\operatorname{Mod}}(\Lambda))}=(-, \underline{G})$.

(ii) Let $F: \overline{\operatorname{Mod}}_{\mathrm{L}}(\Lambda) \rightarrow \mathscr{A} b$ be a homological functor which preserves products and coproducts. Then there exists a finitely presented Gorensteinprojective module $G$ such that $\left.H\right|_{\mathscr{G}_{1}(\overline{\operatorname{Mod}}(\Lambda))}=(\underline{G},-)$. 
Proof. $(\alpha)$ (i) Obviously $H$ restricts to a cohomological functor $\mathscr{G}_{\mathbf{P}}(\underline{\operatorname{Mod}}(\Lambda))^{o p} \rightarrow \mathscr{A} b$, which sends coproducts to products. Then the assertion follows from Brown representability. ( $\alpha$ )(ii) $H$ restricts to a homological functor $\mathscr{G}_{\mathbf{P}}(\underline{\operatorname{Mod}}(\Lambda)) \rightarrow \mathscr{A} b$, which preserves products and coproducts. Then the assertion follows from the above Lemma and [31]. Part $(\beta)$ is treated similarly.

Examples of functors satisfying the condition of $(\alpha)$ (i) in the above Corollary, are the functors $\mathscr{E} x t^{i}(-, A), i \geq 1, \forall A \in \operatorname{Mod}(\Lambda)$. Examples of functors satisfying the condition of $(\alpha)$ (ii) in the above Corollary, are the functors $(F,-), \forall F \in \bmod (\Lambda)$. Examples of functors satisfying the condition of $(\beta)$ (i) in the above Corollary, are the functors $(-, \bar{A}), \forall A \in \operatorname{Mod}(\Lambda)$. Examples of functors satisfying the condition of $(\beta)$ (ii) in the above Corollary, are the functors $\mathscr{E} x t^{i}(F,-), i \geq 1, \forall F \in \bmod (\Lambda)$.

We recall that a full subcategory $\mathscr{L}$ of $\operatorname{Mod}(\Lambda)$ is called resolving, resp. coresolving, if $\mathscr{L}$ is closed under extensions, kernels of epics, resp. cokernels of monics, and contains the projectives, resp. injectives.

COROLlary 6.12. Let $\Lambda$ be a left coherent and right perfect right Gorenstein ring of dimension d. Let $\mathscr{L}$ be a resolving, subcategory of $\operatorname{Mod}(\Lambda)$, closed under coproducts and cokernels of left projective approximations. If $\mathscr{L}$ consists of Gorenstein-projective modules, then $\mathscr{L}$ is contravariantly finite.

Proof. Since $\mathscr{L}$ is resolving, the hypothesis implies that the stable category $\mathscr{L}$ is a localizing subcategory of the compactly generated triangulated category $\mathscr{G}_{\mathbf{P}}(\underline{\operatorname{Mod}}(\Lambda))$. By Corollary 3.18, the inclusion functor $\mathscr{L} \hookrightarrow \mathscr{G}_{\mathbf{P}}(\underline{\operatorname{Mod}}(\Lambda))$ has a right adjoint $R$. Since by [8] the inclusion functor $\mathscr{G}_{\mathbf{P}}(\operatorname{Mod}(\Lambda)) \hookrightarrow$ $\underline{\operatorname{Mod}}(\Lambda)$ has a right adjoint $\Omega^{-d} \Omega^{d}$, it follows that the functor $R \Omega^{-d} \Omega^{d}$ : $\underline{\operatorname{Mod}}(\Lambda) \rightarrow \mathscr{L}$ is a right adjoint of the inclusion $\mathscr{L} \hookrightarrow \operatorname{Mod}(\Lambda)$, hence $\mathscr{L}$ is a coreflective subcategory of $\underline{\operatorname{Mod}}(\Lambda)$. This implies trivially that $\mathscr{L}$ is contravariantly finite in $\operatorname{Mod}(\Lambda)$.

A similar result holds also for coresolving subcategories.

REMARK 6.13. If the ring $\Lambda$ is left coherent right perfect and right Gorenstein, then there exists a skeletally small generating compact left triangulated subcategory in $\underline{\operatorname{Mod}}(\Lambda))$, namely $\underline{\bmod }(\Lambda)$ ), and a compactly generated triangulated category, namely $\mathscr{G}_{\mathbf{P}}(\underline{\operatorname{Mod}}(\Lambda))$, such that the stabilization of $\left.\underline{\bmod }(\Lambda)\right)$ is identified with the full subcategory of compact objects of $\mathscr{G}_{\mathbf{P}}(\operatorname{Mod}(\Lambda))$. This procedure is similar with the construction of the stable homotopy category of spectra in Algebraic Topology and explains our motivation. Here the role of $\underline{\operatorname{Mod}}(\Lambda))$ is played by the homotopy category of spectra, the role of $\underline{\bmod }(\Lambda))$ is played by the homotopy category of finite spectra and the role of $\mathscr{G}_{\mathbf{P}}(\underline{\operatorname{Mod}}(\Lambda))$ 
is played by the stable homotopy category. However in contrast to the homotopy theory of modules, the stable homotopy category of spectra is not the stabilization of all spectra since the latter does not has arbitrary coproducts, so it is not compactly generated, see [32]. Similar remarks are applied for the injective stable category, if $\Lambda$ is right Morita.

\subsection{Phantomless and Brown Stable Homotopy Categories}

Let $\mathscr{C}$ be a compactly generated triangulated category. A morphism $f: A \rightarrow$ $B$ in $\mathscr{C}$ is called phantom if $\mathscr{C}(X, f)=0$, for any $X \in \mathscr{C}^{b}$. The set of all phantom maps between $A, B$ is denoted by $\mathrm{Ph}(A, B)$; setting $\mathrm{Ph}(\mathscr{C})=$ $\bigcup_{A, B \in \mathscr{C}} \mathrm{Ph}(A, B)$ we obtain an ideal in $\mathscr{C}$. We denote by $\operatorname{Ph}^{n}(A, B)$ the set of all morphisms $A \rightarrow B$ which may be written as a composition of $n$ phantom maps and by $\mathrm{Ph}^{n}(\mathscr{C})$ the induced ideal of $\mathscr{C}$. The category $\mathscr{C}$ is called phantomless if $\mathrm{Ph}(\mathscr{C})=0$.

Since $\mathscr{C}^{b}$ is skeletally small, we can consider the Grothendieck category $\operatorname{Mod}\left(\mathscr{C}^{b}\right)$. Since $\mathscr{C}^{b}$ is triangulated, the full subcategory Flat $\left(\operatorname{Mod}\left(\mathscr{C}^{b}\right)\right)$ of flat functors coincides with the category of cohomological functors over $\mathscr{C}^{b}$ [6]. Define a functor

$$
\mathbf{T}: \mathscr{C} \rightarrow \operatorname{Mod}\left(\mathscr{C}^{b}\right) \quad \text { by } \quad \mathbf{T}(A)=\left.\mathscr{C}(-, A)\right|_{\mathscr{C}^{b}}
$$

where $\left.\mathscr{C}(-, A)\right|_{\mathscr{C}^{b}}$ denotes restriction. Then $\mathbf{T}$ is a homological functor with image in Flat $\left(\operatorname{Mod}\left(\mathscr{C}^{b}\right)\right)$ and kernel the ideal $\operatorname{Ph}(\mathscr{C})$ of phantom maps. The category $\mathscr{C}$ is called a Brown category if $\mathbf{T}$ induces a representation equivalence between $\mathscr{C}$ and Flat $\left(\operatorname{Mod}\left(\mathscr{C}^{b}\right)\right)$. We recall that a functor is a representation equivalence if it is full, surjective on objects and reflects isomorphisms.

Definition 6.14. Let $\mathscr{C}$ be a left or right triangulated category with coproducts.

(1) We say that $\mathscr{C}$ admits a phantomless stable homotopy category if $\mathscr{C}$ admits a stable homotopy category $\mathscr{D}$ which is phantomless.

(2) We say that $\mathscr{C}$ admits a Brown stable homotopy category if $\mathscr{C}$ admits a stable homotopy category $\mathscr{D}$ which is a Brown category.

A ring $\Lambda$ has a right injective phantomless, resp. Brown, stable homotopy category if this holds for the right triangulated category $\overline{\operatorname{Mod}}(\Lambda)$. Similarly for the projective case. In this subsection we study rings $\Lambda$ which have injective or projective phantomless or Brown stable homotopy categories. We recall that a ring $\Lambda$ is called representation finite if $\Lambda$ is right Artinian and the set of isomorphism classes of indecomposable finitely presented right modules is finite. It is well known that representation finiteness is a symmetric condition and that $\Lambda$ is representation finite iff $\Lambda$ is left and right pure-semisimple. The 
proof of the following result is a consequence of the results of [6], [7]. For part (3), see also [11].

Proposition 6.15. (1) Let $\Lambda$ be a $Q F$-ring.

(i) $\Lambda$ has a left or right projective or injective phantomless stable homotopy category $\Leftrightarrow \Lambda$ is representation finite.

(i) If $\Lambda$ is countable or $|\Lambda| \leq \aleph_{t}$ for some $t \geq 0$ and $\Lambda$ is pure hereditary (i.e. if the right pure global dimension r.pure.gl.dim $\Lambda \leq 1$ ), then $\Lambda$ has a right projective or injective Brown stable homotopy category.

(2) If $\Lambda$ is a finite dimensional selfinjective local $k$-algebra over an algebraically closed field, then $\Lambda$ has a left and right projective or injective Brown stable homotopy category $\Leftrightarrow \Lambda$ is representation finite or $k$ is countable.

(3) If $G$ is a finite group and $k$ is a field with $\operatorname{char}(k)=p /|G|$, then $k G$ has a left or right projective or injective Brown stable homotopy category $\Leftrightarrow$ $k$ is countable or $G$ has cyclic p-Sylow subgroups.

We note that the stable homotopy category of spectra is a Brown category [32].

Proposition 6.16. Let $\Lambda$ be a ring as in Theorem 6.7. Assume that

$$
\sup \left\{\text { r.pure.p.d. } G ; G \in \mathscr{G}_{\mathbf{P}}(\operatorname{Mod}(\Lambda))\right\}=n<\infty \text {. }
$$

Then $\forall A, B \in \operatorname{Mod}(\Lambda)$ :

$$
\operatorname{Ph}^{n+1}\left(\Omega^{d}(\underline{A}), \Omega^{d}(\underline{B})\right)=0=\operatorname{Ph}^{n+1}\left(\Sigma^{d}(\bar{A}), \Sigma^{d}(\bar{B})\right) .
$$

In particular $\mathrm{Ph}^{n+1}\left(\mathscr{G}_{\mathbf{P}}(\underline{\operatorname{Mod}}(\Lambda))\right)=0=\operatorname{Ph}^{n+1}\left(\mathscr{G}_{\mathbf{I}}(\overline{\operatorname{Mod}}(\Lambda))\right)$.

Proof. This follows from the results of [6], since the hypothesis implies that any object in $\mathscr{G}_{\mathbf{P}}(\underline{\operatorname{Mod}}(\Lambda))$ has a resolution of length $n$ by proper triangles in the sense of relative homological algebra developed in [6].

THEOREM 6.17. Let $\Lambda$ be a right Gorenstein ring. If $\Lambda$ is left coherent and right perfect or right Morita, then the following are equivalent.

(i) $\Lambda$ admits a right projective phantomless stable homotopy category.

(ii) Any Gorenstein-projective is pure projective (pure injective).

(iii) $\mathscr{G}_{\mathbf{P}}(\operatorname{Mod}(\Lambda))$ is a pure-semisimple locally finitely presented Frobenius category.

(iv) $\mathscr{G}_{\mathbf{P}}(\underline{\operatorname{Mod}}(\Lambda))$ is a pure-semisimple locally finitely presented triangulated category.

(v) $\Lambda$ admits a right injective phantomless stable homotopy category. 
(vi) Any Gorenstein-injective is pure injective (pure projective).

(vii) $\mathscr{G}_{\mathbf{I}}(\operatorname{Mod}(\Lambda))$ is a pure-semisimple locally finitely presented Frobenius category.

(viii) $\mathscr{G}_{\mathbf{I}}(\overline{\operatorname{Mod}}(\Lambda))$ is a pure-semisimple locally finitely presented triangulated category.

Proof. Assume first that $\Lambda$ is left coherent and right perfect ring. If condition (i) holds, then $\mathscr{G}_{\mathbf{P}}(\operatorname{Mod}(\Lambda))$ is phantomless. By [6], we have that any object $\underline{G} \in \mathscr{G}_{\mathbf{P}}(\underline{\operatorname{Mod}}(\Lambda))$ is a coproduct $\oplus_{i \in I} \underline{G}_{i}$, where $\underline{G}_{i}$ is in $\mathscr{G}_{\mathbf{P}}(\underline{\bmod }(\Lambda))$. But then in $\operatorname{Mod}(\Lambda)$ we have $G=\oplus_{i \in I} G_{i} \oplus P$, where $P$ is projective. Since $\Lambda$ is right perfect, this implies that $G$ is a coproduct of finitely presented Gorenstein modules. Hence $G$ is in particular pure-projective. Hence (i) $\Rightarrow$ (ii). If (ii) holds, then obviously $\mathscr{G}_{\mathbf{P}}(\underline{\operatorname{Mod}}(\Lambda))=\operatorname{Add}\left(\mathscr{G}_{\mathscr{P}}(\underline{\bmod }(\Lambda))\right)$. Since $\mathscr{G}_{\mathscr{P}}(\underline{\bmod }(\Lambda))$ is skeletally small and is contained in $\mathscr{G}_{\mathbf{P}}(\operatorname{Mod}(\Lambda))^{b}$, by Proposition 2.5 it follows that $\mathscr{G}_{\mathbf{P}}(\operatorname{Mod}(\Lambda))$ is a pure-semisimple locally finitely presented category. Hence (ii) $\Rightarrow$ (iii). If (iii) holds, then any Gorensteinprojective module is a coproduct of finitely presented (Gorenstein-projective) modules, and trivially the same is true in $\mathscr{G}_{\mathbf{P}}(\underline{\operatorname{Mod}}(\Lambda))$. By [6], $\mathscr{G}_{\mathbf{P}}(\underline{\operatorname{Mod}}(\Lambda))$ is a locally finitely presented (phantomless) pure-semisimple triangulated category and this shows that (iii) $\Rightarrow$ (iv). Since by [6] any locally finitely presented triangulated category is phantomless, we have that (iv) $\Rightarrow$ (i). Now we prove that any of the above equivalent conditions (i) - (iv) is equivalent to the parenthetical condition (ii). We use that an object $G$ in a locally finitely presented category with products is pure-injective iff for any index set $I$, the summation map $f: \oplus_{i \in I} G \rightarrow G$ factors through the pure mono $\mu_{I}: \oplus_{i \in I} G \rightarrow \prod_{i \in I} G$, see [13]. Suppose now that (i) is true and let $G$ be a Gorenstein-projective module. Let $I$ be any index set and consider the summation map $f: \oplus_{i \in I} G \rightarrow G$. Since $\mathscr{G}_{\mathbf{P}}(\underline{\operatorname{Mod}}(\Lambda))$ is a pure semisimple locally finitely presented category, the morphism $\underline{f}: \oplus_{i \in I} \underline{G} \rightarrow \underline{G}$ factors through the canonical morphism $\mu: \oplus_{i \in I} \underline{G} \rightarrow \prod_{i \in I} \underline{G}$ : there exists a morphism $\underline{\alpha}: \prod_{i \in I} \underline{G} \rightarrow \underline{G}$ such that $f=\mu \circ \underline{\alpha}$. Then there exist a morphism $\beta: \oplus_{i \in I} G \rightarrow P$ such that $f-\mu \circ \alpha=\beta \circ p$, where $p: P \rightarrow G$ is the projective cover of $G$. Since $P$ is projective and $\Lambda$ is right perfect, $P$ is pure-injective. Since $\mu: \bigoplus_{i \in I} G \rightarrow \prod_{i \in I} G$ is a pure mono, there exists a morphism $t: \prod_{i \in I} G \rightarrow P$ such that $\mu \circ t=\beta$. Then $f=\mu \circ(\alpha+t \circ p)$. This shows that the summation map $f$ factors through the pure mono $\mu$. Hence $G$ is pure-injective. Conversely if any Gorenstein-projective module $G$ is pure-injective, then using the same argument as above, it follows that all objects of $\mathscr{G}_{\mathbf{P}}(\underline{\operatorname{Mod}}(\Lambda))$ are pure-injective in the sense of [31]. Hence by [31], $\mathscr{G}_{\mathbf{P}}(\underline{\operatorname{Mod}}(\Lambda))$ is phantomless.

Since $\mathscr{G}_{\mathbf{P}}(\underline{\operatorname{Mod}}(\Lambda))$ is triangle equivalent to $\mathscr{G}_{\mathbf{P}}(\overline{\operatorname{Mod}}(\Lambda))$, using the same 
arguments as above we have that the remaining conditions are also equivalent to (i). If $\Lambda$ is right Morita, the proof is similar and is left to the reader.

From the (proof of the) above Theorem we deduce the following results.

CoRollary 6.18. Let $\Lambda$ be a left coherent and right perfect or right Morita right Gorenstein ring. Then $\Lambda$ admits a phantomless projective or injective stable homotopy category iff any Gorenstein-projective or Gorensteininjective module is a coproduct of finitely presented Gorenstein-projective or Gorenstein-injective modules with local endomorphism rings. In this case the coproduct decomposition is essentially unique.

COROLlaRY 6.19. Let $\Lambda$ be a right pure-semisimple right Gorenstein ring. If $\Lambda$ is left coherent or right Morita, then $\Lambda$ has a right projective and injective phantomless stable homotopy category. In particular this happens if $\Lambda$ is a representation-finite Artin algebra of finite selfinjective dimension.

We close this section studying rings with Brown stable homotopy category.

THEOREM 6.20. Let $\Lambda$ be a right Gorenstein ring of dimension d. Suppose that $|\Lambda| \leq \aleph_{t}$, for some $t \geq-1$ and $\Lambda$ is left coherent and right perfect or right Morita.

If r r.pure.p.d $A \leq 1$, for any Gorenstein-projective module $A$ or r.pure.i.d $B$ $\leq 1$, for any Gorenstein-injective module $B$, then $\Lambda$ has a right projective and injective Brown stable homotopy category.

Proof. Since the Brown property is invariant under triangle equivalence, it suffices to prove the projective case. By the results of [7], it suffices to show that for any object $\underline{G}$ in $\mathscr{G}_{\mathbf{P}}(\underline{\operatorname{Mod}}(\Lambda))$, there exists a triangle $\underline{G}_{1} \rightarrow \underline{G}_{0} \rightarrow$ $\underline{G} \stackrel{h}{\rightarrow} \Omega^{-1}\left(\underline{G}_{1}\right)$ in $\mathscr{G}_{\mathbf{P}}(\underline{\operatorname{Mod}}(\Lambda))$, where $\underline{h}$ is a phantom map and $\underline{G}_{1}, \underline{G}_{0} \in$ $\operatorname{Add}\left(\mathscr{G}_{\mathscr{P}}(\underline{\bmod }(\Lambda))\right)$. Let $(*): 0 \rightarrow X_{1} \stackrel{\alpha}{\rightarrow} X_{0} \stackrel{\beta}{\rightarrow} G \rightarrow 0$ be a pure-projective resolution of the Gorenstein-projective module $G$. Then we have a triangle $\Omega(\underline{G}) \rightarrow \underline{X}_{1} \stackrel{\alpha}{\rightarrow} \underline{X}_{0} \stackrel{\beta}{\rightarrow} \underline{G}$ in $\underline{\operatorname{Mod}}(\Lambda)$. Applying the stabilization functor $\mathbf{S}=\Omega^{-d} \Omega^{d}$ to this triangle, we obtain a triangle $\mathbf{S}\left(\underline{X}_{1}\right) \stackrel{\mathbf{S}(\underline{\alpha})}{\longrightarrow} \mathbf{S}\left(\underline{X}_{0}\right) \stackrel{\mathbf{S}(\underline{\beta})}{\longrightarrow}$ $\underline{G} \stackrel{h}{\rightarrow} \Omega^{-1} \mathbf{S}\left(\underline{X}_{1}\right)$ in $\left.\mathscr{G}_{\mathbf{P}}(\underline{\operatorname{Mod}}(\Lambda))\right)$. Since the stabilization functor preserves coproducts and $X_{0}, X_{1}$ are pure projectives it follows that $\mathbf{S}\left(\underline{X}_{0}\right), \mathbf{S}\left(\underline{X}_{1}\right) \in$ $\operatorname{Add}\left(\mathscr{G}_{\mathscr{P}}(\underline{\bmod }(\Lambda))\right)$. We remains to prove that $\underline{h}$ is phantom. Let $\underline{H} \in$ $\mathscr{G}_{\mathscr{P}}(\underline{\bmod }(\Lambda))$ and $g: \underline{H} \rightarrow \underline{G}$ be a morphism. Since $H$ is finitely presented, there exists a morphism $\delta: H \rightarrow X_{0}$ such that $\delta \circ \beta=g$. Then $\mathbf{S}(\underline{\delta}) \circ \mathbf{S}(\underline{\beta})=g$. This shows that $\underline{h}$ is phantom.

COROLLARY 6.21. Let $\Lambda$ be a left coherent and right perfect right Gorenstein ring of dimension d. Then $\Lambda$ admits a Brown right projective stable homotopy 
category iff for any filtered directed system of finitely presented right modules $\left\{A_{i} ; \phi_{i j}\right\}$, there exists a minimal weak colimit $\mathrm{w} . \lim _{\rightarrow} \Omega^{d}\left(\underline{A}_{i}\right)$ in $\mathscr{G}_{\mathscr{P}}(\underline{\operatorname{Mod}}(\Lambda))$, i.e. $\lim _{\rightarrow}\left(\underline{G}, \Omega^{d} \underline{A}_{i}\right) \cong\left(\underline{G}, \mathrm{w} . \lim _{\rightarrow} \Omega^{d}\left(\underline{A}_{i}\right)\right), \forall G \in \mathscr{G}_{\mathscr{P}}(\bmod (\Lambda))$. In this case the following are true:

(1) $\forall A \in \operatorname{Mod}(\Lambda)$, the object $\Omega^{d}(\underline{A})$ is a minimal weak colimit $\Omega^{d}(\underline{A})=$ w. $\lim \underline{G}_{i}$ of a directed system $\left\{\underline{G}_{i} ; i \in I\right\}$ in $\mathscr{G}_{\mathscr{P}}(\bmod (\Lambda))$.

(2) If $A, B \in \operatorname{Mod}(\Lambda)$ and $\Omega^{d}(\underline{A})=\mathrm{w} \cdot \lim \underline{G}_{i}, \Omega^{d}(\underline{B})=\mathrm{w} \cdot \lim \underline{H}_{i}$ are representations as minimal weak colimits as $\overrightarrow{a b o v e, ~ t h e n ~ t h e r e ~ e x i s t s ~ a ~ s h o r t ~}$ exact sequence

$0 \rightarrow \lim _{\leftarrow}^{(1)} \lim _{\rightarrow}\left(\underline{G}_{i}, \Omega\left(\underline{H}_{j}\right)\right) \rightarrow\left(\Omega^{d}(\underline{A}), \Omega^{d}(\underline{B})\right) \rightarrow \lim _{\leftarrow} \lim _{J}\left(\underline{G}_{i}, \underline{H}_{j}\right) \rightarrow 0$

and isomorphisms:

$$
\begin{gathered}
\operatorname{Ph}\left(\Omega^{d}(\underline{A}), \Omega^{d}(\underline{B})\right) \cong \lim _{\leftarrow}^{(1)} \lim _{J}\left(\underline{G}_{i}, \Omega\left(\underline{H}_{j}\right)\right) \\
\cong \lim _{I}^{(1)} \lim _{J} \mathscr{E} x t^{1}\left(G_{i}, \Omega^{2}\left(H_{j}\right)\right) .
\end{gathered}
$$

(3) $\forall A, B \in \operatorname{Mod}(\Lambda)$, we have:

$$
\mathrm{Ph}^{2}\left(\Omega^{d}(\underline{A}), \Omega^{d}(\underline{B})\right)=0=\mathrm{Ph}^{2}\left(\Sigma^{d}(\bar{A}), \Sigma^{d}(\bar{B})\right) .
$$

Proof. All the assertions are consequences of the results of [7].

A similar result is true for right Morita right Gorenstein rings.

Corollary 6.22. If $\Lambda$ is a countable Artin algebra of finite self-injective dimension, then $\Lambda$ has a right projective and injective Brown stable homotopy category.

We refer to [6], [7] for further consequences of the phantomless or Brown property of projective or injective stable homotopy categories.

ACKNOWLEDGEMENTS. The author gratefully acknowledges support from the EC TMR Network "Orbits, Crystals and Representation Theory", under the contract No. FMRX-CT97-0100. This work was done during a stay at the University of Bielefeld. The author expresses his gratitude to the members of the Representation Theory Group at Bielefeld, and especially to Prof. Dr. C. M. Ringel, for the warm hospitality. Finally the author thanks Prof. T. Pirashvili, for bringing to his attention the results of the paper [34].

Note ADDED IN PROOF. The preprints [6] and [7] have appeared in Relative homological algebra and purity in triangulated categories, J. Algebra 227 (2000), 268-361. 


\section{REFERENCES}

1. Auslander, M. and Bridger, M., Stable Module Theory, Mem. Amer. Math. Soc. 94 (1969).

2. Auslander, M. and Smalø, S. O., Preprojective modules over Artin algebras, J. Algebra 66 (1980), 61-122.

3. Auslander, M. and Reiten, I., Cohen-Macaulay and Gorenstein Artin algebras, Prog. Math. 95 (1991), 221-245.

4. Beilinson, A., Bernstein, J. and Deligne, P., Faisceaux Pervers, Astérisque 100 (1982).

5. Beligiannis, A., On The Freyd categories of an additive category, Homology, Homotopy and Applications 2 (No. 11) (2000), 147-185.

6. Beligiannis, A., Homological algebra in triangulated categories I: Relative homology and phantomless triangulated categories, Preprint, UNAM, Mexico, 1997.

7. Beligiannis, A., Homological algebra in triangulated categories II: Representation theorems, localization and the stable category, Preprint, UNAM, Mexico, 1997.

8. Beligiannis, A., The homological theory of contravariantly finite subcategories, Comm. Algebra 28 (10) (2000), 4547-4596.

9. Beligiannis, A. and Marmaridis, N., Left triangulated categories arising from contravariantly finite subcategories, Comm. Algebra 22 (1994), 5021-5036.

10. Beligiannis, A. and Marmaridis, N., Grothendieck groups arising from contravariantly finite subcategories, Comm. Algebra 24 (1996), 4415-4438.

11. Benson, D. J. and Gnacadja, G. PH., Phantom maps and purity in modular representation theory I, Fund. Math. 161 (1999), 37-91.

12. Brown, E. H., Abstract homotopy theory, Trans. Amer. Math. Soc. 119 (1965), 79-85.

13. Crawley-Boevey, W., Locally finitely presented additive categories, Comm. Algebra 22, (1994), 1644-1674.

14. Dwyer, W. G. and Spalinski, J., Homotopy theories and model categories, I. M. James (Ed), Handbook of Algebraic Topology, North-Holland, 1995, 73-126.

15. Eckmann, B. and Kleisli, H., Algebraic homotopy groups and Frobenius algebras, Illinois J. Math. 6 (1962), 533-552.

16. Freyd, P., Splitting homotopy idempotents, in: Proceedings of the Conference on Categorical Algebra, La Jolla, (1966), 173-176.

17. Gabriel, P. and Zisman, M., Calculus of Fractions and Homotopy Theory, Springer Verlag, New York, (1967).

18. Heller, A., The loop space functor in homological algebra, Trans. Amer. Math. Soc. 96 (1960), 382-394.

19. Heller, A., Stable homotopy categories, Bull. Amer. Math. Soc. 74 (1968), $28-63$.

20. Heller, A., Completions in abstract homotopy theory, Trans. Amer. Math. Soc. 147 (1970), 573-602.

21. Hilton, P. J., Homotopy Theory and Duality, Gordon and Breach, New York, 1965.

22. Hovey, M., Model Categories, Math. Surveys Monographs 63, 1999.

23. Huber, P., Homotopy theory in general categories, Math. Ann. 144 (1961), 361-385.

24. Jensen, C. U. and Simson, D., Purity and generalized chain conditions, J. Pure Appl. Algebra 14 (1979), 297-305.

25. Jørgensen, P., Brown representability for stable categories, Math. Scand. 85 (1999), 195-218.

26. Keller, B., Deriving DG categories, Ann. Sci. École Norm. Sup. 27 (1994), 63-102.

27. Keller, B. and Vossieck, D., Sous les cateégories dérivées, C. R. Acad. Sci. Paris 305 (1987), 225-228.

28. Keller, B. and Vossieck, D., Aisles in derived categories, Bull. Soc. Math. Belg. 40 (1988), 239-253.

29. Kleisli, H., Homotopy theory in abelian categories, Canad. J. Math. 14 (1962), 139-169. 
30. Krause, H., Functors on locally finitely presented categories, Colloq. Math. 75 (1998), 105132.

31. Krause, H., Smashing subcategories and the telescope conjecture - An algebraic approach, Invent. Math. 139 (No. 1) (2000), 99-133.

32. Margolis, H. R., Spectra and the Steenrod Algebra, North-Holland Math. Library 29, 1983.

33. Neeman, A., The Grothendieck duality theorem via Bousfield's techniques and Brown representability, J. Amer. Math. Soc. 9 (1996), 205-236.

34. Pirashvili, T., Closed model category structures on abelian categories (in Georgian), Bull. Georgian Acadamy of Sciences (= Soobsch. Akad. Nauk Gruzin. SSR) 124 (1986), 265268.

35. Quillen, D., Homotopical Algebra, Lecture Notes in Math. 43 (1967).

36. Rickard, J., Idempotent modules in the stable module category, J. London Math. Soc. 178 (1997), 149-170.

37. Verdier, J. L., Catégories derivées, état 0, Lecture Notes in Math. 569 (1977), 262-311.

38. Waldhausen, F., Algebraic K-theory of spaces, Lecture Notes in Math. 1126 (1985), 318-419.

FAKULTÄT FÜR MATHEMATIK

UNIVERSITÄT BIELEFELD

D-33501 BIELEFELD

GERMANY

E-mail: abelig@mathematik.uni-bielefeld.de
CURRENT ADDRESS:

DEPARTMENT OF MATHEMATICS

UNIVERSITY OF THE AEGEAN

83200 KARLOVASSI

GREECE

E-mail: abeligia@mail.otenet.gr 